\title{
Long-term effects of network-based fMRI neurofeedback training for sustained attention
}

Gustavo S.P. Pamplona ${ }^{1,2,3,4, *}$, Jennifer Heldner ${ }^{3}$, Robert Langner ${ }^{5,6}$, Yury Koush $^{7}$, Lars Michels ${ }^{8,9}$, Silvio Ionta ${ }^{1}$, Carlos E.G. Salmon ${ }^{2, * *}$, Frank Scharnowski $i^{3,9,10,11, * *}$

1. Sensory-Motor Laboratory (SeMoLa), Jules-Gonin Eye Hospital/Fondation Asile des Aveugles, Department of Ophthalmology/University of Lausanne, Lausanne, Switzerland

2. InBrain Lab, Department of Physics, University of Sao Paulo, Ribeirao Preto, Brazil

3. Department of Psychiatry, Psychotherapy and Psychosomatics, Psychiatric Hospital, University of Zurich, Switzerland

4. Rehabilitation Engineering Laboratory (RELab), Department of Health Sciences and Technology, ETH Zurich, Zurich, Switzerland

5. Institute of Systems Neuroscience, Heinrich Heine University Dusseldorf, Dusseldorf, Germany

6. Institute of Neuroscience and Medicine, Brain \& Behaviour (INM-7), Research Centre Julich, Julich, Germany

7. Department of Radiology and Biomedical Imaging, Yale School of Medicine, Yale University, New Haven, CT, USA

8. Department of Neuroradiology, University Hospital Zurich, Zurich, Switzerland

9. Neuroscience Center Zurich, University of Zurich and Swiss Federal Institute of Technology, Zurich, Switzerland

10. Zurich Center for Integrative Human Physiology (ZIHP), University of Zurich, Zurich, Switzerland

11. Department of Cognition, Emotion, and Methods in Psychology, Faculty of Psychology, University of Vienna, Vienna, Austria

*Corresponding author: gsppamplona@gmail.com

** Both authors contributed equally. 


\section{ABSTRACT}

Neurofeedback allows for learning voluntary control over one's own brain activity, aiming to enhance cognition and clinical symptoms. A recent study improved sustained attention temporarily by training healthy participants to up-regulate the differential activity of the sustained attention network (SAN) minus the default mode network (DMN). However, long-term learning effects of functional magnetic resonance imaging (fMRI) neurofeedback training remain underexplored. Here, we evaluate the effects of network-based fMRI neurofeedback training for sustained attention by assessing behavioral and brain measures before, one day after, and two months after training. The behavioral measures include task as well as questionnaire scores, and the brain measures include activity and connectivity during self-regulation runs without feedback (i.e., transfer runs) and during resting-state runs. Neurally, we found that participants maintained their ability to control the differential activity during follow-up sessions. Also, the training-induced increase in FC between the DMN and occipital gyrus was maintained during follow-up transfer runs. In contrast, the training-induced increased FC between DMN and occipital gyrus was not observed during follow-up resting-state runs. Behaviorally, we found that enhanced sustained attention right after training returned to baseline level during follow-up. The discrepancy between lasting regulation-related brain changes but transient behavioral and resting-state effects raises the question of how neural changes induced by neurofeedback training translate to potential behavioral improvements. Since neurofeedback directly targets brain measures to indirectly improve behavior long-term, a better understanding of the brain-behavior associations during and after neurofeedback training is needed to develop its full potential as a promising scientific and clinical tool. 


\section{INTRODUCTION}

Neurofeedback is a form of biofeedback that provides individuals with real-time sensory information about their own brain activity, over which voluntary control can be learned with training (Sitaram et al., 2017). This technique has gained interest to be translated as a clinical intervention for addressing neuropsychological diseases (Bu et al., 2019; Linden et al., 2012; Zweerings et al., 2019). This is because learned brain self-regulation has been frequently associated with cognitive restoration and sustained positive clinical effects (Sitaram et al., 2017; Sulzer et al., 2013a). In healthy individuals, neurofeedback has been shown to bolster mental functions (Amano et al., 2016; deBettencourt et al., 2015; Pamplona et al., 2020a), typically characterized by concomitant neural plasticity (Megumi et al., 2015; Scheinost et al., 2013; Yuan et al., 2014; Zhang et al., 2013). Functional magnetic resonance imaging (fMRI) neurofeedback provides high spatial resolution and whole-brain coverage, therefore offering the targeting of specific brain regions and high flexibility in modelling and computing feedback signals (Amano et al., 2016; Koush et al., 2017b; Scheinost et al., 2020; Watanabe et al., 2017). Recently, some fMRIneurofeedback studies also addressed the training of large-scale brain networks (Kim et al., 2019; Krause et al., 2021; Pamplona et al., 2020a), accounting for complex cognitive functions that are better represented by the interaction among intercommunicating regions of the brain than by single-region-based models.

Although transient behavioral effects and the mitigation of clinical symptoms through fMRIneurofeedback intervention are of high neuroscientific relevance, the long-term persistence of training outcomes is important for the consolidation of the technique as a useful tool for improving cognitive abilities and promoting recovery. Of particular interest, long-lasting neuroplasticity induced by neurofeedback may support the modulation of the neural mechanism underlying the transferred behavioral effect or clinical improvement. Several fMRI-neurofeedback studies evaluated the long-term persistence of acquired neural modulations in runs with or without feedback (Ramot et al., 2017; Robineau et al., 2017), behavioral learning effects measured by psychometric techniques or questionnaires (Amano et al., 2016; Mehler et al., 2018; Zilverstand et al., 2015), and changes in functional connectivity (FC) (Harmelech et al., 2013; Megumi et al., 2015). It was proposed that fMRI-neurofeedback may not only lead to persistent effects but might even trigger continuing improvement of effects over days and even months after training completion (Rance et al., 2018). However, neurofeedback studies vary considerably in terms of induced immediate learning effects due to the variability of experimental designs, individual capacity to train specific brain activity and FC neurofeedback, target cognitive functions and brain 
regions, feedback signal estimation and data processing approaches (Fede et al., 2020; Harmelech et al., 2015; Haugg et al., 2020; Sulzer et al., 2013a). Due to the high variability of factors influencing immediate neurofeedback learning effects, long-term learning effects may also vary. Systematic investigation of long-term neurofeedback learning effects could help understand learning mechanisms and facilitate therapeutic translation of neurofeedback training.

We recently demonstrated that sustained attention for a limited time period can be improved using fMRI neurofeedback of simultaneous up-regulation of the sustained attention network (SAN) and down-regulation of the default-mode network (DMN) (Pamplona et al., 2020a). We found that participants of the neurofeedback group showed an improvement of sustained attention directly after training. This was not observed in the test-retest control group, which performed the sustained attention tests, similarly to the neurofeedback group, but did not train neurofeedback. We hypothesized that neural and behavioral changes induced by sustained attention training would persist or even continue to improve in the longer term. To test this hypothesis, we comprehensively characterized previously unpublished data from resting-state runs and from twomonths after neurofeedback training session. We examined the translation of trained neurofeedback regulation to situations without neurofeedback, using regulation-specific (regFC) and resting-state (rSFC) functional connectivity in transfer and resting-state runs, respectively, as well as behavioral scores, measured before, directly after and two months after the neurofeedback training. Here, we used a multidimensional approach to evaluate effects induced by the neurofeedback training in the long term. Namely, we investigated (i) changes in pre-training self-regulated activation of SAN and DMN and other brain regions two months after neurofeedback training, to characterize the sustained ability to control activity without feedback; (ii) changes in pre-training regFC and rsFC directly after neurofeedback training and two months later, in order to investigate sustained neuroplasticity with and without voluntary regulation; (iii) changes in the degree of rSFC of trained regions of the SAN and DMN directly after neurofeedback training and two months later, to understand long-term plastic specificity due to training; and (iv) changes in training-induced attentional aspects measured by tests and questionnaires two months after neurofeedback training, to evaluate the sustaining of behavioral effects. 


\section{METHODS}

\subsection{Participants}

We included data from a previously published study (Pamplona et al., 2020a). In the neurofeedback group, participants performed the neurofeedback training. In the control group, participants performed the sustained attention tests twice (separated by a two-week interval, similarly as for the neurofeedback group) without neurofeedback training. In the present study, we analyzed data only from the neurofeedback group, composed of 15 healthy volunteers (5 females, Mean = 27.9, S.D. = 3.3, range $=[22.6,34.5]$ years old). Data included behavioral tests, transfer and resting-state runs before, directly after and two months after neurofeedback training. Exclusion criteria were left-handedness, strong vision deficiency that could not be corrected by the use of contact lenses, insufficient knowledge of English, history of mental and/or cardiovascular disorders, not being able to abstain from alcohol or other drugs during the days of experiment, and MRI contraindications. This study was approved by the local ethics committee of the Canton of Zurich in Switzerland. All participants read and signed the informed consent according to the Declaration of Helsinki 2013 before taking part in the study. They received financial compensation of $25 \mathrm{CHF}$ per hour for their participation.

\subsection{Experimental procedure}

Each participant took part in a five-day longitudinal study (Fig. 1) that involved fMRIneurofeedback training and pre/post-training sessions. The neurofeedback training consisted of ten real-time fMRI runs spanned over two days (Fig. 1, 5 runs per day). After the last neurofeedback training session, we included a post-training (one day after training) and a longterm follow-up (two months after training, $61 \pm 3$ days) sessions. Neurofeedback training runs consisted of five cycles of baseline, regulation, and intermittent feedback blocks, lasting $30 \mathrm{~s}, 40$ $\mathrm{s}$, and $4 \mathrm{~s}$, respectively. To signalize the period of baseline, regulation, and feedback blocks, participants were presented with a black square, a black up-arrow, and a graded thermometer on the center of a white screen, respectively.

For the neurofeedback group, we evaluated post-training and long-term effects of neurofeedback training through transfer runs, resting-state runs, and psychometric tests and questionnaires. Transfer runs are used to verify neurofeedback training transfer effects in situations when the feedback is withdrawn. We acquired single transfer runs before and after training and two 2-month 
follow-up transfer runs (Megumi et al., 2015). For a thorough evaluation of long-term neurofeedback effects, we also estimated FC changes during transfer and resting-state fMRI runs before, after and 2-months after training. To evaluate follow-up continuity and neurofeedbackinduced alterations in behavioral states, participants performed psychometric tests outside the MR scanner before, after and 2-months after training.

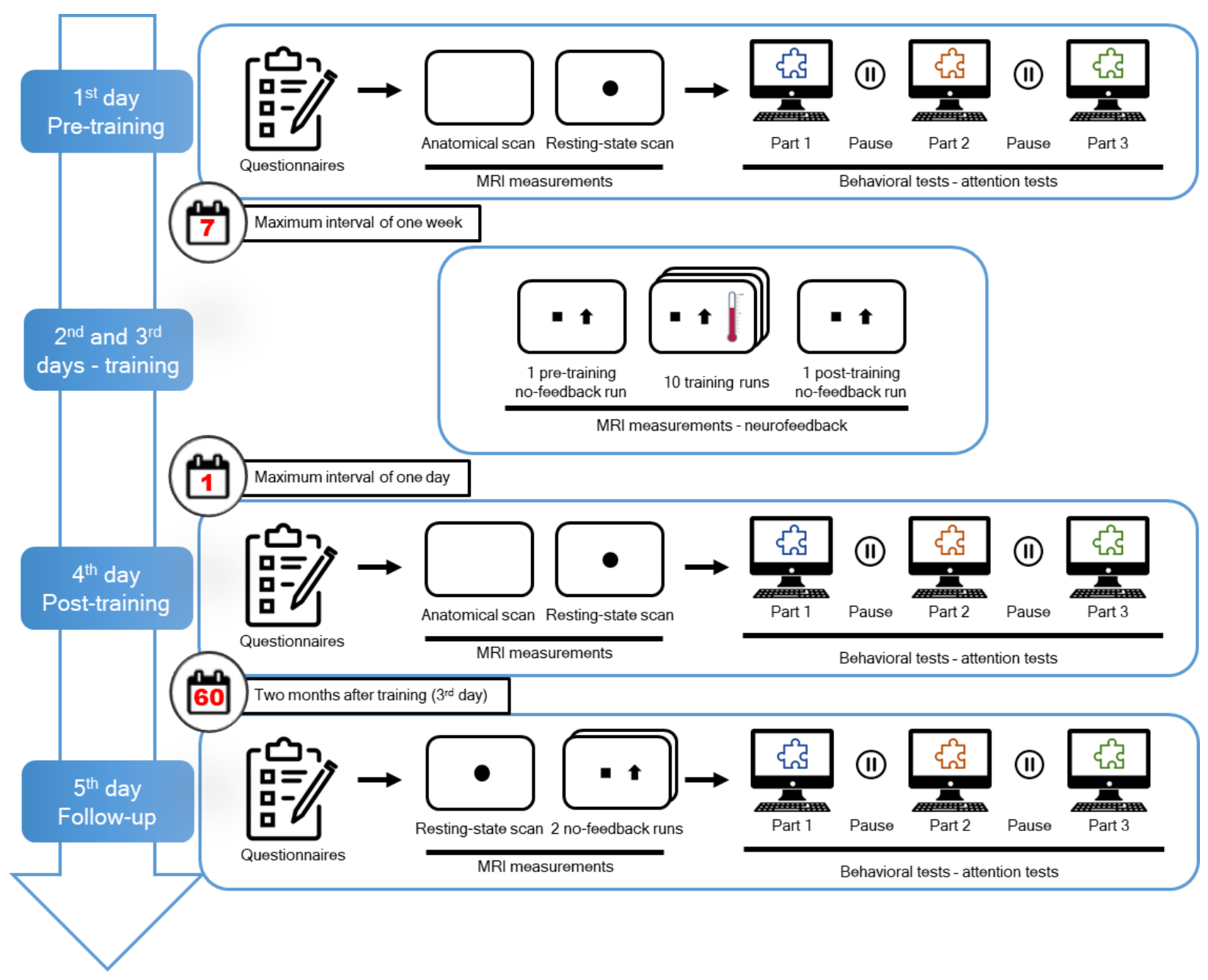

Figure 1. Timeline of the five-day neurofeedback experimental procedure. On the first, fourth, and fifth days of experiment, participants filled out attention-related questionnaires (DSSQ and CFQ), performed a resting-state $\mathrm{fMRI}$ acquisition and attention-related tests on a computer (blue puzzle piece: Continuous Performance Task and Switcher; orange puzzle piece: PVT and Mental Rotation Task; green puzzle piece: Attentional Network Test). Additionally, participants performed two transfer regulation runs on the fifth day. The neurofeedback training sessions occurred on the second and third days of the experiment. 
Experimental sessions were performed with a maximum of one week separating the first and the second day, one day separating the third and fourth days, and two months separating the third and the fifth days.

Instructions. Before each neurofeedback session, instructions for self-regulation strategies were provided in written form. We instructed the participants to relax and let their minds wander during baseline blocks and to engage in a suggested or in a self-idealized attention-related strategy (e.g., focusing on the black up-arrow and bringing the attention back to it whenever task-unrelated thoughts were detected) during regulation blocks. Same instructions were provided for the pretraining training transfer run. For post-training and follow-up transfer runs, participants were asked to use the strategy that worked best during the neurofeedback training.

Data acquisition. We acquired functional and anatomical MR images using a Philips Achieva 3T MRI scanner and 32-channel head coil. Transfer (180 scans, 6:00 total duration) and resting-state (200 scans, 6:40 total duration) functional runs were acquired using gradient-echo $\mathrm{fMRI}$ sequence (repetition time/echo time $=2000 / 30 \mathrm{~ms}$, voxel size $=3 \times 3 \times 4 \mathrm{~mm}^{3}$, gap $=0.5 \mathrm{~mm}$, 37 slices, flip angle $=80^{\circ}$ ). During resting-state acquisitions, we additionally asked participants to fix their gaze on a small black circle at the center of a white screen in a room with dimmed lights and let the mind wander freely. During the entire study course, participants were asked to avoid movements, stay relaxed, and breathe regularly (for further data acquisition details, refer to Pamplona et al., 2020a).

Definitions of target networks. Sustained attention is a cognitive function that supports the continuous focus on a particular external object for longer periods. Sustained attention is anatomically non-unitary and rather comprises a set of regions whose activation is related to distinct processes underpinning externally-oriented attention and its maintenance (Langner and Eickhoff, 2013). In contrast, the activation of the DMN is related to internally focused cognitive processes and mind-wandering (Andrews-Hanna et al., 2014; Raichle et al., 2001); when performing an externally-oriented task, the DMN activation is related to lower attentional performance and attentional lapses (Hinds et al., 2011; Lawrence et al., 2003; Thompson et al., 2013; Weissman et al., 2006). These two networks are hence inherently anticorrelated, as they are engaged in antagonistic processes reflecting externally- versus internally-oriented attention (Fox et al., 2005; Spreng, 2012). To improve sustained attention using neurofeedback training, we therefore addressed this cognitive function by training the dominance of the sustained attention network (SAN) over the default mode network (DMN), positively and negatively associated with sustained attention performance, respectively. To define the SAN, we used a 
sustained attention meta-analysis study (Langner and Eickhoff, 2013) (Table S1). The SAN comprised the anterior mid-cingulate cortex (related to conflict processing, monitoring performance, and enhanced vigilance (Hinds et al., 2013; Langner and Eickhoff, 2013; Weissman et al., 2006)), the right inferior frontal junction (related to stimuli discrimination and switching attention (Langner and Eickhoff, 2013)), the right temporoparietal junction (bottom-up reorientation of attention (Corbetta and Shulman, 2002; Weissman et al., 2006)), and the right intraparietal sulcus (right IPS - top-down reorienting of attention (Corbetta and Shulman, 2002; Harris et al., 2000). The DMN was defined by using subject-specific resting-state acquisitions, the independent component analysis (ICA) approach as implemented in Gift (mialab.mrn.org/software/gift), and the individualization of region definition as implemented in Personode toolbox (Pamplona et al., 2020b; www.nitrc.org/projects/personode) (Table S1). The DMN comprised the posterior cingulate cortex (PCC), the medial prefrontal cortex (mPFC), and the bilateral angular gyri.

Feedback estimation and presentation. During feedback blocks, the difference between normalized average signals from the SAN and DMN (differential activity SAN minus DMN), considering previous regulation and baseline blocks, was fed back to the participant as the level of the thermometer. Participants were asked to raise the thermometer level as much as possible, which could be achieved either by SAN upregulation, DMN downregulation, or both. Real-time fMRI data were acquired and processed using OpenNFT (Koush et al., 2017a). Feedback presentation was adaptive for each run based on the performance in previous runs, i.e., the feedback was made harder to achieve if the task was relatively easy to the participant and viceversa. At the end of each run, a monetary reward was given to the participant proportional to their performance in the current run (CHF $20.6 \pm 5.4$ in total per participant; for further feedback estimation and presentation details, see Pamplona et al., 2020a).

\subsection{Behavioral tests and questionnaires}

To assess changes in different aspects of attention due to the neurofeedback training, participants performed a battery of five executive-attention-related tests implemented in the Psychology Experiment Building Language software (Mueller and Piper, 2014) (Fig. 1). The battery comprised of the Continuous Performance Task (Conners et al., 2003), the Task-Switching Performance (Switcher) (Anderson, et al., 2012), the Psychomotor Vigilance Test (PVT) (Dinges and Powell, 1985), the Mental Rotation Task (Shepard and Metzler, 1971), and the Attentional Network Test (Fan et al., 2002) (for further procedural information, refer to (Pamplona et al., 2020a)). To assess 
long-term neurofeedback-induced changes in attention, we used only PVT metrics. This is because in our first publication we found that only changes related to PVT were associated with the neurofeedback training. The PVT assesses how fast participants respond to unexpected stimuli, inferring on the individual sustained attention capability. Written instructions were provided before each test application.

To evaluate long-term changes in attentional states and traits, participants also completed attention-related questionnaires across sessions [Dundee State Questionnaire (DSSQ) (Helton, 2004) and Cognitive Failure Questionnaire (CFQ) (Broadbent et al., 1982), respectively]. Technical failures in the acquisition led to incomplete questionnaire data collection. Specific DSSQ sub-scores were computed using only part of the dataset (inclusion of 14-15 participants in the pre-training session, 6-7 participants in the post-training session, and 12-15 participants in the follow-up session - number of participants varies depending on the sub-score).

To evaluate changes in the subjectively perceived state of concentration (i.e., self-evaluated sustained attention) and adopted regulation strategies applied during the transfer runs, we asked the participants to report them right after each run. In addition, at the end of each transfer run, participants rated their level of concentration in the previous run on a scale ranging from 1 (very low) to 10 (very high). Due to technical issues with the communication system, we were not able to collect the self-report concentration ratings from two participants.

\subsection{Data analysis}

fMRI preprocessing. Transfer and resting-state functional images as well the anatomical images were preprocessed using SPM12 (www.fil.ion.ucl.ac.uk) in MATLAB (The MathWorks, Natick, MA, USA). First, functional images were slice-time corrected using the middle slice as reference. Then, three translation and three rotation parameters of head movement were estimated, and functional images were spatially realigned to a created mean functional image. Next, the anatomical image was coregistered to the mean functional image and then segmented into tissue probability masks for gray matter, white matter, and cerebrospinal fluid (CSF) compartments. During the process of segmentation, a deformation field was created, which was used to normalize the anatomical and functional images to the standard MNI space. Finally, the normalized transfer functional images were spatially smoothed using a Gaussian kernel of $8 \mathrm{~mm}$ full-width at half maximum (FWHM) and the normalized resting-state functional images were smoothed with a 6-mm kernel FWHM. 


\subsubsection{Transfer run activity and FC analyses}

First-level analysis for activation. In the neurofeedback group, we investigate differences in the training-induced neural activity across pre-, post-training and follow-up transfer runs. For firstlevel analysis, we specified the general linear model (GLM) with two regressors of interest for two conditions (i.e., separately for regulation and baseline conditions) and six head motion covariates per run. Regressors of interest were modelled with boxcar functions convolved with the canonical hemodynamic response function, as implemented in SPM12. Next, betas (regression weights) of regulation and baseline blocks for each participant and each run were estimated voxel-wise. Contrast for activation differences between regulation and baseline blocks were created for each participant and run.

Long-term effects on regulation in target networks. We compared average beta coefficients from SAN and DMN networks and from their encompassing brain areas across transfer runs. Beta coefficients of regulation and baseline regressors were averaged within SAN, DMN and within each predefined brain area of these networks using MarsBaR (marsbar.sourceforge.net, Brett, Anton, Valabregue, \& Poline, 2002). In order to account for practice effects in follow-up transfer runs (as the subjects returned to the experimental session after a long time), the follow-up runs were analyzed separately in this analysis. We compared the average signal SAN minus DMN between each of the two follow-up runs and the pre-training runs with paired t-tests or Wilcoxon signed-rank tests, depending on parametric or non-parametric distributions, respectively. These tests were performed with RStudio (www.rstudio.com). These comparisons were also performed for SAN and DMN separately, as well as for each of the four constituent brain areas per network. Normality of each session-specific distribution of variables was evaluated through Shapiro-Wilk tests. Consistent with the direction of the expected changes after neurofeedback training (i.e., more positive activity for the difference SAN minus DMN and for SAN and more negative activity for DMN), these pairwise tests were one-tailed.

Whole-brain long-term effects on regulation. First, individual contrasts were entered into a second-level analysis in which subjects were treated as random effects. One-sample t-tests against the zero-level at each voxel were performed for each session (i.e., pre-training, posttraining, and follow-up) to map activations and deactivations for the group. Additionally, we also created individual contrasts comparing post- versus pre-training sessions and comparing followup (i.e., averaged across runs) versus pre-training sessions. Beta coefficients of two separate 
follow-up runs were averaged. These contrasts were treated as random effects in second-level analyses with one-sample t-tests (which is equivalent to a paired t-test with partitioned errors (Henson, 2015)). All resulting second-level maps were submitted to the threshold-free cluster estimation (TFCE) approach with 10000 permutations and a voxel-level threshold of $p<0.001$ uncorrected for multiple comparisons. This approach provides high sensitivity in detecting both large and small clusters (Smith and Nichols, 2009) and is a suitable alternative for non-normal distributions or low sample sizes. These thresholded maps were anatomically labelled by the bspmview toolbox (www.bobspunt.com/software/bspmview/; Spunt, 2016).

regFC changes across transfer runs. We used psychophysiological interaction (PPI) analysis to investigate changes in the FC of the target networks with the whole-brain, modulated by the periods of regulation versus baseline during transfer runs (McLaren et al., 2012; O'Reilly et al., 2012). This analysis was performed using the toolbox CONN (version 19.c) (Whitfield-Gabrieli and Nieto-Castanon, 2012). Seed-based PPI maps were estimated using a standard two-level analysis across pre-training, post-training, and follow-up sessions. For the first-level analysis, blocks of regulation and baseline were defined as regressors of interest. Seeds were the four regions comprising SAN and the four subject-specific regions comprising DMN targeted in the neurofeedback training. These SAN and DMN regions were masked with subject-specific gray matter maps. Regressors of no-interest were defined as the six realignment parameters, their first-level derivatives, and five principal components from white matter and CSF time-series (Behzadi, Restom, Liau, \& Liu, 2007). Additional denoising to the voxelwise time-series included bandpass filtering $(0.008$ to $0.09 \mathrm{~Hz})$, despiking, and linear detrending. The seed-based regFC contrast (regulation versus baseline) was estimated voxelwise for each participant using a GLM. For the second-level analysis, contrast images from all participants were entered into a Wilks' Lambda test, a multivariate approach alternative to the repeated-measures ANOVA with increased robustness to the violation of the compound-symmetry assumption, and the withinsubject variance was inferred across pre-training, post-training, and follow-up sessions. Thresholded statistical t-value maps were generated using the Gaussian random-field theory (Worsley et al., 1996) with a cluster-level threshold of $p<0.05$, FWE (family-wise error)-corrected for multiple comparisons, and a voxel-level inclusion threshold of $p<0.001$. Post-hoc analyses were performed to determine pairwise differences across sessions using the library 'emmeans' in RStudio according to a $p<0.05$, Tukey-corrected for multiple comparisons. FC clusters were anatomically labelled with xjView (www.alivelearn.net/xjview). 


\subsection{2. rsFC analyses}

Seed-based rsFC. We used rsFC to investigate changes in the FC of the target networks with the whole brain at rest due to neurofeedback training. This rsFC analysis was performed using the CONN toolbox. SAN and DMN seed-based rsFC maps were estimated using a two-level analysis across pre-training, post-training, and follow-up sessions. For the first-level analysis, the seedbased rsFC was estimated voxelwise for each participant. Seeds were the regions comprising SAN and DMN targeted in the neurofeedback training, masked with subject-specific gray matter maps. The regressors of no-interest were defined similarly as in the regFC analysis. Additional denoising included bandpass filtering, despiking, and linear detrending. For the second-level analysis, FC images from all participants were entered into a Wilks' Lambda test and the withinsubject variance was inferred across sessions. Thresholded statistical t-value maps were generated using the Gaussian random-field theory with a cluster-level threshold of $p<0.05$, FWEcorrected for multiple comparisons, and a voxel-level inclusion threshold of $p<0.001$. Post-hoc analyses were performed to determine pairwise differences across sessions $(p<0.05)$. FC clusters were anatomically labelled with xjView.

Changes in the degree of rSFC. We investigated modulations in the degree of rsFC within regions that are part of SAN and DMN across pre-training, post-training, and follow-up sessions. In graph theory applied to neuroimaging, the degree is defined as the number of edges of an individual node in a given network and threshold (Rubinov and Sporns, 2010). The degree of FC estimates to which extent a target network region is connected with the whole brain. Here, the degree of rsFC was computed using the intrinsic connectivity distribution (ICD) approach (Scheinost et al., 2012), which does not require the choice of an arbitrary threshold. Specifically for this analysis, slice-time corrected and realigned resting-state functional images were first normalized and resampled to a voxel size of $4 \times 4 \times 4 \mathrm{~mm}^{3}$ to reduce computational load in ICD computation and smoothed using a kernel of $8 \mathrm{~mm}$ FWHM. The ICD was computed for each voxel, participant, and session using a customized code as reported in (Scheinost et al., 2012). To investigate changes in the degree of SAN and DMN regions, we averaged the ICD values within these regions for each participant and session. One-way repeated-measures ANOVAs were computed for each SAN and DMN region (4 regions per network), in which session was defined as the within-subject factor. Post-hoc analyses were performed in RStudio using the library 'emmeans'. p-values were adjusted for multiple comparisons at the region level by the Tukey method. We estimated effect sizes for the main effect and the pairwise comparisons, i.e., $\eta^{2}$ and Cohen's $d$, respectively. 


\subsubsection{Analysis of behavioral effects}

We investigated changes in the ability of sustained attention across sessions, measured by the PVT. We previously reported that sustained attention improved for a limited period due to our neurofeedback training (Pamplona et al., 2020a). Here, we tested whether this improvement persisted in the follow-up session. PVT outcomes are the reaction times to the stimuli over time; each repetition consisting of a trial. Here, we analyzed differences of reaction time in both early and late trials of the test. We used linear mixed models to account for the hierarchical structure (multiple measurements of response time for each subject), with the factors Session and Trial; Trial being a continuous variable. As we were interested in differences of reaction time over trials across sessions, we checked whether the two-way interaction Session $\mathrm{x}$ Trial was significant. We then proceeded by post-hoc comparing main effects of session in the reaction time in early and late trials separately, defined here as one standard deviation minus the average trial and one standard deviation plus the average trial, respectively, according to the standard convention (Cohen and Cohen, 1983; West et al., 1996). Subject is defined as a random factor, Session and Trial were defined as fixed factors. For the linear mixed model and post-hoc analyses, we used the libraries 'Ime4' and 'emmeans' in RStudio ( $p$-values adjusted for multiple comparisons using the Tukey method), respectively. We also investigated changes in self-reported attention, namely the DSSQ sub-scores and the CFQ, between follow-up and pre-training sessions (the difference post- minus pre-training was already computed in the previous publication). We used paired ttests and dependent two-group Wilcoxon signed-rank tests, depending on the normality of the distributions, respectively, as assessed by Shapiro-Wilk tests. We hypothesized some DSSQ subscores to be modulated across sessions (motivation, self-focused attention, concentration, control and confidence, task-related interference), but we also performed exploratory analyses of attentional states and traits (all possible sub-scores from DSSQ and CFQ). The p-values were corrected for multiple comparisons using false discovery rate (FDR) correction for the number of scores in each analysis. 


\section{RESULTS}

\subsection{Follow-up effects during regulation without feedback}

3.1.1. Long-term effects on regulation in target networks

Extending our reported findings (Pamplona et al., 2020a), the previously learned regulation of the differential activity SAN minus DMN without feedback was present after two months (paired ttests between pre-training and follow-up runs: first follow-up run: $t(14)=1.92, p=0.04$; second follow-up run: $\mathrm{t}(10)=2.05, \mathrm{p}=0.03$ ) (Fig. $2 \mathrm{~A}$ ). This long-lasting effect was mainly driven by $a$ persistent down-regulation of the DMN as an entity (paired t-tests between pre-training and followup runs, first follow-up run: $\mathrm{t}(14)=2.05, \mathrm{p}=0.03$; second follow-up run: $\mathrm{t}(10)=1.76, \mathrm{p}=0.054$ ) (Fig. 2B). We did not detect changes in the up-regulation performance of the SAN as a whole compared to pre-training levels (paired t-tests between pre-training and follow-up runs; first followup run: $\mathrm{t}(14)=0.22, \mathrm{p}=0.4$; second follow-up run: $\mathrm{t}(10)=0.91, \mathrm{p}=0.19)$ (Fig. S1A).
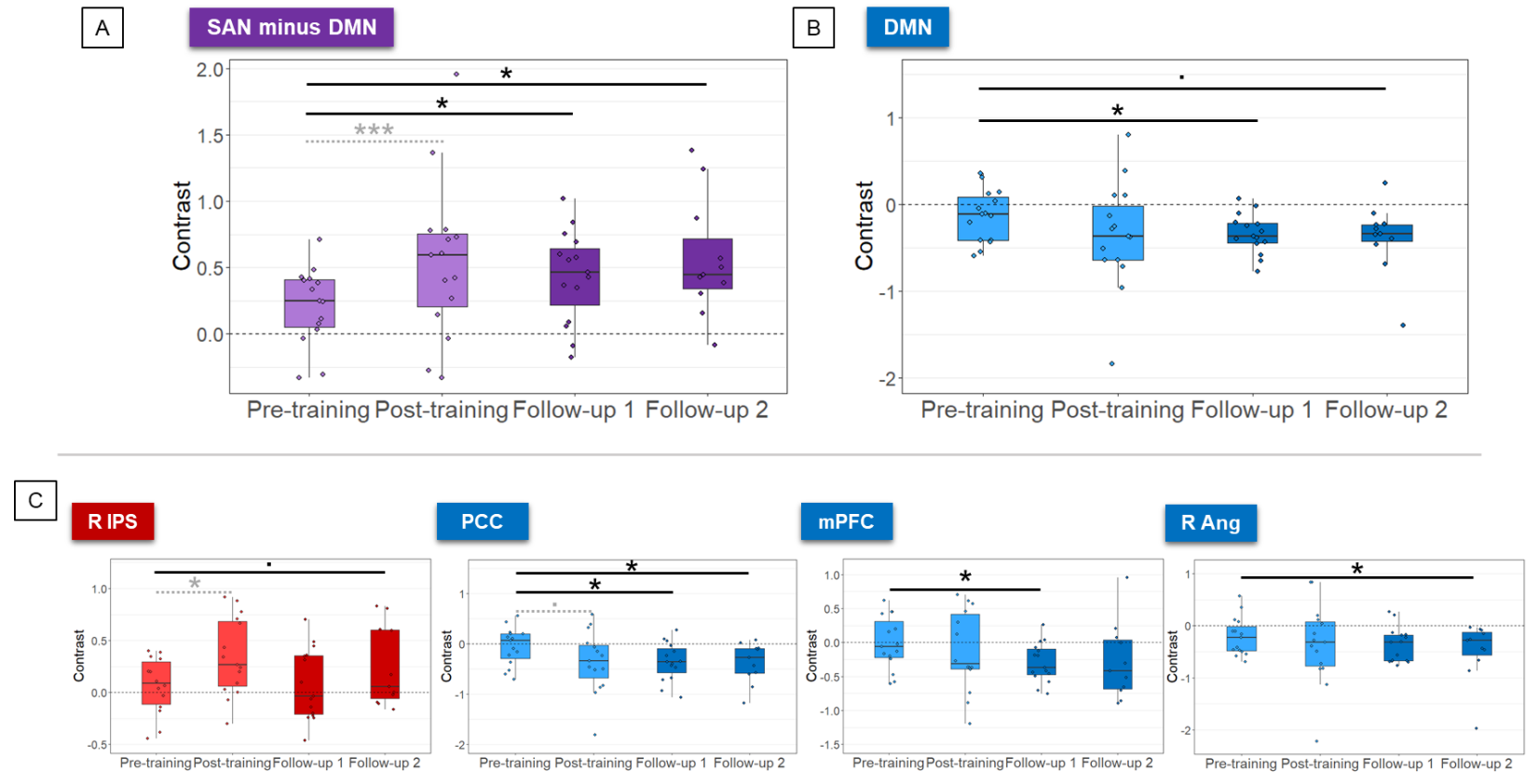

Figure 2. Increased network self-regulation was present two months after the end of neurofeedback training (A), which was driven by an augmented ability in down-regulating the DMN (B). Specifically, differences in self-regulation ability were observed in individual target regions of the DMN, as well as in the right IPS (part of the SAN) (C). The plots show the contrast of the activation levels between regulation and baseline blocks for pre-training, post-training, and the two follow-up transfer acquisitions. Purple, blue, and red colors represent the differential activity SAN minus DMN, DMN, and SAN regions, respectively. Bright and dull 
colors represent follow-up and pre-/post-training sessions, respectively. The gray dashed line represents a significant difference previously reported in (Pamplona et al., 2020a). Asterisks represent significant session differences $\left({ }^{* *} p<00001,{ }^{*} p<0.05, . p<0.06\right.$, uncorrected).

When analyzing separately the regulation of individual target SAN and DMN regions over transfer runs, we observed that the learned ability of up-regulating right IPS and down-regulating PCC was maintained in the follow-up runs, respectively (right IPS: second follow-up run > pre-training, $\mathrm{V}=52, \mathrm{p}=0.051$; PCC: first follow-up run < pre-training, $\mathrm{t}(14)=2.31, \mathrm{p}=0.018$, second followup run < post-training, $\mathrm{t}(10)=2.25, \mathrm{p}=0.025$ ) (Fig. $2 \mathrm{C}$ ). In addition, we found that the ability of down-regulating mPFC and right angular gyrus improved only in the follow-up but not in the posttraining session (mPFC: first follow-up run < pre-training, $\mathrm{t}(14)=2.08, \mathrm{p}=0.028$; right angular gyrus: second follow-up run < post-training, $\mathrm{V}=12, \mathrm{p}=0.03$ ) (Fig. $2 \mathrm{C}$ ). We observed no differences in other SAN and DMN regions (Fig. S1B).

\subsubsection{Whole-brain long-term effects on regulation}

Whole-brain analyses showed significant deactivation (i.e., activation in the regulation period < baseline) clusters in the DMN during the follow-up transfer session (i.e., averaged over the two follow-up runs) (Fig. 3 and Table 1). Significant clusters showing deactivation in the PCC and mPFC were larger in the follow-up compared to the post-training session. Activation in the DAN was detected in all transfer sessions. The thalamus was activated in the follow-up session. A complete list of activated and deactivated clusters is reported in Table 1. The contrasts postminus pre-training and follow-up minus pre-training showed decreased activity in the left and right middle occipital gyrus, respectively, for the follow-up and the post-training compared to pretraining session (Fig. S2 and Table 1). 
bioRxiv preprint doi: https://doi.org/10.1101/2021.10.27.465722; this version posted November 15, 2021. The copyright holder for this preprint

(which was not certified by peer review) is the author/funder, who has granted bioRxiv a license to display the preprint in perpetuity. It is made available under aCC-BY-ND 4.0 International license.

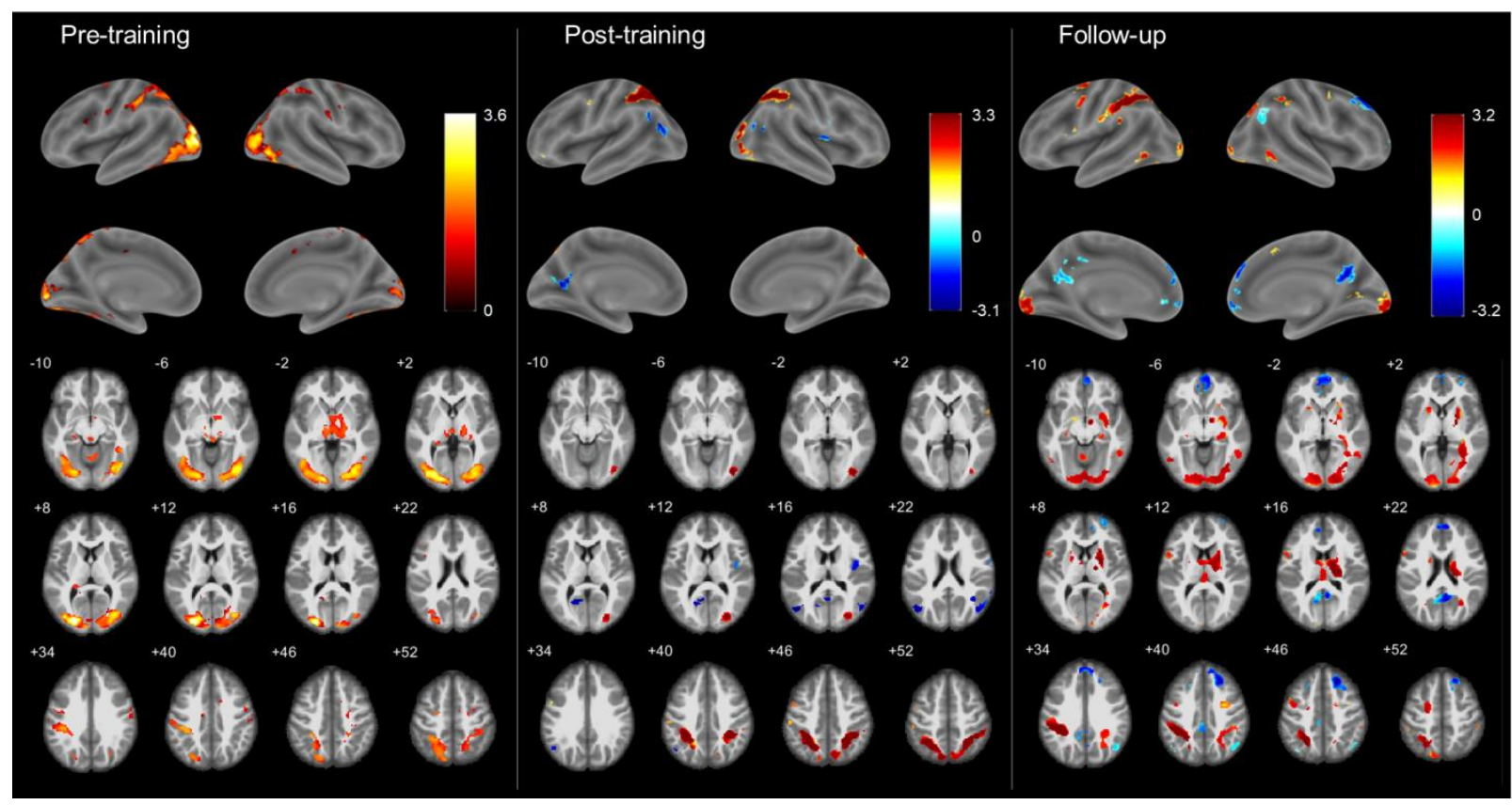

Figure 3. Whole-brain analyses show enhanced ability in down-regulating the DMN in the follow-up transfer sessions, compared to pre- and post-training sessions. The dorsal attention network was activated across all sessions. The thalamus was observed in the follow-up session. Left, middle, and right columns show pre-training, post-training, and follow-up sessions, respectively. Hot and cold colors represent significant activation and deactivation clusters during regulation blocks compared to baseline blocks, respectively, overlapped onto surface-rendered (top) and axial slices (bottom) from a brain template. T-maps were generated by TFCE, thresholded at $p<0.001$ uncorrected.

Table 1. Significant positive and negative activations during pre-, post-training, and follow-up sessions, as shown in Figures 3, and contrasts for post- minus pre-training session and follow-up minus pre-training session, as shown in Figure S2. Brain regions represent local maxima peak separated by more than $20 \mathrm{~mm}$. Only clusters with more than 20 voxels are shown. $L / R / C=$ left/right/center.

\begin{tabular}{|c|c|c|c|c|c|c|c|}
\hline \multirow{2}{*}{$\begin{array}{l}\text { Activation } \\
\text { direction }\end{array}$} & \multirow{2}{*}{ Region Label } & \multirow{2}{*}{ Laterality } & \multirow{2}{*}{ Extent } & \multirow{2}{*}{$\begin{array}{l}\text { Cluster } \\
\text { peak t- } \\
\text { value }\end{array}$} & \multicolumn{3}{|c|}{$\begin{array}{l}\text { Cluster peak MNI } \\
\text { coordinates }\end{array}$} \\
\hline & & & & & $\mathrm{x}$ & y & $z$ \\
\hline \multicolumn{8}{|c|}{ Pre-training session } \\
\hline \multirow{14}{*}{ Positive } & Middle Occipital Gyrus, Inferior Temporal Gyrus & $\mathrm{L}$ & 4629 & 3.56 & -24 & -90 & 6 \\
\hline & Inferior Occipital Gyrus, Fusiform Gyrus, Inferior Temporal Gyrus & $\mathrm{R}$ & 3560 & 3.45 & 36 & -76 & -6 \\
\hline & Superior Frontal Gyrus & $\mathrm{L}$ & 84 & 3.30 & -20 & -4 & 60 \\
\hline & Caudate Nucleus & $\mathrm{R}$ & 596 & 3.30 & 18 & -20 & 0 \\
\hline & Superior Parietal Lobule, Postcentral Gyrus & $\mathrm{R}$ & 616 & 3.26 & 26 & -40 & 48 \\
\hline & White matter & $\mathrm{C}$ & 46 & 3.25 & 2 & -36 & -6 \\
\hline & Paracentral Lobule, Posterior-Medial Frontal & $\mathrm{C}$ & 114 & 3.24 & -6 & -38 & 70 \\
\hline & Cerebellum (VI) & $\mathrm{L}$ & 51 & 3.23 & -18 & -60 & -20 \\
\hline & Precentral Gyrus & $\mathrm{L}$ & 71 & 3.22 & -42 & -4 & 32 \\
\hline & Hippocampus & $\mathrm{L}$ & 50 & 3.19 & -22 & -38 & 6 \\
\hline & White matter & $\mathrm{R}$ & 24 & 3.18 & 26 & -28 & 2 \\
\hline & White matter & $\mathrm{R}$ & 30 & 3.16 & 24 & -14 & 50 \\
\hline & Inferior Frontal Gyrus (p. Triangularis) & $\mathrm{L}$ & 100 & 3.14 & -44 & 20 & 28 \\
\hline & Precentral Gyrus & $\mathrm{R}$ & 105 & 3.13 & 58 & -2 & 36 \\
\hline \multicolumn{8}{|c|}{ Post-training session } \\
\hline \multirow{3}{*}{ Positive } & Mid Orbital Gyrus & $\mathrm{R}$ & 152 & 3.06 & 26 & 42 & -18 \\
\hline & Inferior Occipital Gyrus & $\mathrm{R}$ & 220 & 3.06 & 40 & -82 & -8 \\
\hline & Middle Occipital Gyrus & $\mathrm{R}$ & 205 & 3.06 & 28 & -84 & 8 \\
\hline
\end{tabular}


bioRxiv preprint doi: https://doi.org/10.1101/2021.10.27.465722; this version posted November 15, 2021. The copyright holder for this preprint (which was not certified by peer review) is the author/funder, who has granted bioRxiv a license to display the preprint in perpetuity. It is made available under aCC-BY-ND 4.0 International license.

\begin{tabular}{|c|c|c|c|c|c|c|c|}
\hline & Middle Occipital Gyrus, Precuneus & $\mathrm{L}$ & 1604 & 3.06 & -32 & -44 & 36 \\
\hline & Inferior Parietal Lobule & $\mathrm{R}$ & 1519 & 3.06 & 36 & -44 & 38 \\
\hline & Mid Orbital Gyrus & $\mathrm{L}$ & 54 & 2.88 & -24 & 50 & -14 \\
\hline & Precentral Gyrus & $\mathrm{L}$ & 32 & 2.83 & -48 & -4 & 48 \\
\hline & Supramarginal Gyrus & $\mathrm{L}$ & 55 & 2.77 & -54 & -30 & 48 \\
\hline \multirow{5}{*}{ Negative } & Middle Temporal Gyrus & $\mathrm{L}$ & 271 & 3.06 & -46 & -74 & 20 \\
\hline & Lingual Gyrus & $\mathrm{L}$ & 144 & 3.04 & -12 & -58 & 8 \\
\hline & Middle Temporal Gyrus & $\bar{R}$ & 233 & 3.01 & 48 & -72 & 18 \\
\hline & Rolandic Operculum & $\mathrm{R}$ & 90 & 2.98 & 42 & -8 & 18 \\
\hline & Rolandic Operculum & $\mathrm{R}$ & 24 & 2.77 & 62 & 0 & 20 \\
\hline \multicolumn{8}{|c|}{ Follow-up } \\
\hline \multirow{19}{*}{ Positive } & Thalamus, Putamen & $\mathrm{R}$ & 1526 & 3.22 & 16 & -14 & 14 \\
\hline & Supramarginal Gyrus, Middle Occipital Gyrus & $\mathrm{L}$ & 1302 & 3.22 & -30 & -44 & 32 \\
\hline & Fusiform Gyrus, Lingual Gyrus & $\mathrm{R}$ & 4028 & 3.18 & 30 & -52 & 2 \\
\hline & Superior Frontal Gyrus & $\mathrm{L}$ & 327 & 3.16 & -24 & -12 & 52 \\
\hline & Superior Parietal Lobule & $L$ & 137 & 3.15 & -30 & -62 & 64 \\
\hline & Inferior Temporal Gyrus & $\mathrm{L}$ & 50 & 3.13 & -50 & -64 & -6 \\
\hline & Precentral Gyrus & $\mathrm{L}$ & 97 & 3.11 & -44 & -4 & 46 \\
\hline & Supramarginal Gyrus & $\mathrm{R}$ & 665 & 3.11 & 24 & -54 & 40 \\
\hline & White matter & $\mathrm{C}$ & 168 & 3.11 & 0 & -22 & 16 \\
\hline & Putamen & $\mathrm{L}$ & 106 & 3.09 & -22 & 0 & 8 \\
\hline & White matter & $\mathrm{L}$ & 28 & 3.09 & -28 & -66 & 26 \\
\hline & Inferior Temporal Gyrus & $\mathrm{R}$ & 147 & 3.08 & 52 & -52 & -6 \\
\hline & Caudate & $\mathrm{L}$ & 27 & 3.07 & -16 & 0 & 18 \\
\hline & Superior Parietal Lobule, Precuneus & $\mathrm{R}$ & 120 & 3.05 & 32 & -60 & 62 \\
\hline & Precuneus & C & 119 & 3.05 & -8 & -80 & 52 \\
\hline & Inferior Frontal Gyrus (p. Opercularis) & $\mathrm{L}$ & 127 & 3.05 & -54 & 10 & 12 \\
\hline & Cingulate Gyrus & $\mathrm{R}$ & 24 & 3.04 & 16 & -4 & 48 \\
\hline & White matter & $\mathrm{R}$ & 93 & 3.00 & 28 & 2 & 40 \\
\hline & Postcentral Gyrus & $\mathrm{L}$ & 34 & 2.99 & -50 & -38 & 56 \\
\hline \multirow{8}{*}{ Negative } & Superior Frontal Gyrus, Superior Medial Gyrus & $\mathrm{R}$ & 1032 & 3.22 & 20 & 34 & 46 \\
\hline & Precuneus & $\mathrm{C}$ & 563 & 3.15 & 6 & -58 & 22 \\
\hline & Mid Orbital Gyrus, Mid Orbital Gyrus & C & 443 & 3.06 & 6 & 60 & -6 \\
\hline & Posterior Cingulate Cortex & C & 152 & 2.98 & 2 & -42 & 36 \\
\hline & Middle Frontal Gyrus & $\mathrm{R}$ & 140 & 2.93 & 34 & 60 & -2 \\
\hline & Middle Frontal Gyrus & $\mathrm{L}$ & 30 & 2.87 & -24 & 28 & 44 \\
\hline & Angular Gyrus & $\mathrm{R}$ & 244 & 2.80 & 44 & -66 & 34 \\
\hline & Angular Gyrus & $\mathrm{L}$ & 23 & 2.53 & -46 & -68 & 42 \\
\hline \multicolumn{8}{|c|}{ Post- minus } \\
\hline Negative & Middle Occipital Gyrus & $\mathrm{L}$ & 75 & 3.06 & -34 & -86 & 8 \\
\hline \multicolumn{8}{|c|}{ Follow-up minus pre-training } \\
\hline \multirow{2}{*}{ Negative } & Middle Occipital Gyrus & $\mathrm{R}$ & 53 & 2.87 & 40 & -78 & 8 \\
\hline & Inferior Occipital Gyrus & $\mathrm{R}$ & 29 & 2.60 & 44 & -70 & -6 \\
\hline
\end{tabular}

\subsection{3. regFC changes across transfer runs}

Significant regFC changes were found across sessions mainly between DMN regions and the left middle occipital gyrus (Fig. 4). For the SAN (seed region), regFC changes are shown in Fig. S3. A summary of significant regFC is shown in Table 2. 
bioRxiv preprint doi: https://doi.org/10.1101/2021.10.27.465722; this version posted November 15,2021 . The copyright holder for this preprint (which was not certified by peer review) is the author/funder, who has granted bioRxiv a license to display the preprint in perpetuity. It is made available under aCC-BY-ND 4.0 International license.

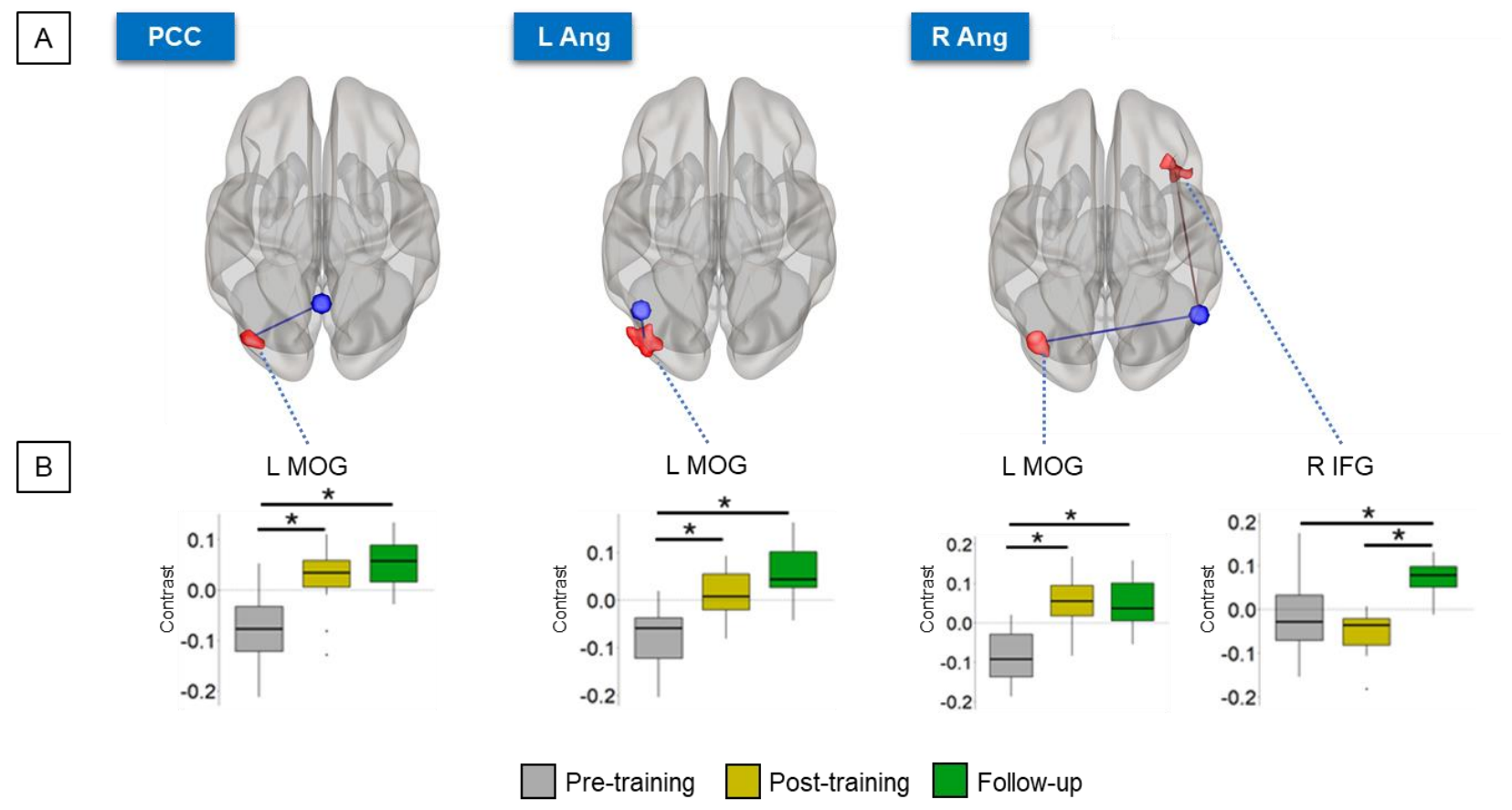

Figure 4. (A) The regFC analyses showed that some clusters (e.g., left middle occipital gyrus) presented differences of FC with individual DMN regions across sessions, considering regulation versus baseline. Blue and red clusters represent DMN regions and seed-to-voxel significant FC clusters, respectively, projected onto glass brains in superior view. (B) Boxplots represent contrast values (regulation versus baseline) from the regFC for each session; gray, yellow, and green represent pre-training, post-training, and follow-up sessions, respectively. The dashed black lines in the boxplots represent the zero level. Asterisks represent significant differences corrected for multiple comparisons by the Tukey method $(p<0.05) . M O G=$ middle occipital gyrus, IFG = inferior frontal gyrus, $L / R=$ left/right .

Table 2. Changes in regulation-specific (regFC) and resting-state ( $r S F C$ ) functional connectivity between SAN/DMN and other clusters as illustrated in Figs. S3 and 4 and 5. A summary of the results from post-hoc analyses of the three contrasts (follow-up minus pre-training, post-training minus pre-training, and follow-up minus post-training) is shown. Blank cells indicate non-significant contrasts.

\begin{tabular}{|c|c|c|c|c|c|c|c|c|c|c|c|c|}
\hline \multicolumn{13}{|c|}{ regFC } \\
\hline \multirow[b]{2}{*}{ Seed } & \multicolumn{2}{|c|}{ Connecting cluster } & \multirow{2}{*}{$\begin{array}{l}\text { Extent } \\
\text { (voxels) }\end{array}$} & \multicolumn{3}{|c|}{ Follow-up minus pre-training } & \multicolumn{3}{|c|}{$\begin{array}{l}\text { Post-training minus pre- } \\
\text { training }\end{array}$} & \multicolumn{3}{|c|}{ Follow-up minus post-training } \\
\hline & Label & $\begin{array}{l}\text { MNI peak } \\
\text { coordinates } \\
(\mathrm{mm})\end{array}$ & & $\mathrm{t}(28)$ & $\begin{array}{l}\text { Cohen's } \\
\text { d }\end{array}$ & p-value & $t(28)$ & $\begin{array}{l}\text { Cohen's } \\
\text { d }\end{array}$ & $p$-value & $\mathrm{t}(28)$ & $\begin{array}{l}\text { Cohen's } \\
\text { d }\end{array}$ & $\mathrm{p}$-value \\
\hline SAN1 & $\begin{array}{l}\text { Right angular } \\
\text { gyrus }\end{array}$ & $(34,-66,44)$ & 120 & +5.38 & +2.16 & $<0.0001$ & - & - & - & +3.97 & +1.29 & 0.0013 \\
\hline SAN2 & $\begin{array}{l}\text { Left } \\
\text { postcentral } \\
\text { gyrus }\end{array}$ & $(-44,-30,54)$ & 142 & -4.13 & -1.41 & 0.0008 & - & - & - & -5.73 & -2.29 & $<0.0001$ \\
\hline SAN3 & $\begin{array}{l}\text { Left } \\
\text { hippocampus }\end{array}$ & $(-18,-8,-20)$ & 133 & +4.86 & +2.00 & 0.0001 & - & - & - & +4.66 & +1.68 & 0.0002 \\
\hline SAN4 & $\begin{array}{l}\text { Left } \\
\text { postcentral } \\
\text { gyrus }\end{array}$ & $(-44,-16,48)$ & 125 & -3.16 & -1.31 & 0.010 & +2.50 & +1.00 & 0.05 & -5.66 & -2.46 & 0.0001 \\
\hline DMN1 & $\begin{array}{l}\text { Left middle } \\
\text { occipital gyrus }\end{array}$ & $(-42,-82,12)$ & 121 & +4.20 & +1.52 & 0.0007 & +5.44 & +2.14 & $<0.0001$ & - & - & - \\
\hline
\end{tabular}


bioRxiv preprint doi: https://doi.org/10.1101/2021.10.27.465722; this version posted November 15,2021 . The copyright holder for this preprint (which was not certified by peer review) is the author/funder, who has granted bioRxiv a license to display the preprint in perpetuity. It is made available under aCC-BY-ND 4.0 International license.

\begin{tabular}{|c|c|c|c|c|c|c|c|c|c|c|c|c|}
\hline DMN3 & $\begin{array}{l}\text { Left middle } \\
\text { occipital gyrus }\end{array}$ & $(-46,-82,8)$ & 165 & +5.83 & +2.09 & $<0.0001$ & +3.97 & +1.53 & 0.0013 & - & - & - \\
\hline \multirow{2}{*}{ DMN4 } & $\begin{array}{l}\text { Left middle } \\
\text { occipital gyrus }\end{array}$ & $(-40,-76,10)$ & 113 & +4.79 & +2.09 & $<0.0001$ & +4.79 & +2.08 & $<0.0001$ & - & - & - \\
\hline & $\begin{array}{l}\text { Right inferior } \\
\text { frontal gyrus }\end{array}$ & $(36,24,-16)$ & 136 & +4.11 & +1.45 & 0.0009 & - & & - & +5.76 & +2.69 & $<0.0001$ \\
\hline \multicolumn{13}{|c|}{ rsFC } \\
\hline DMN4 & $\begin{array}{l}\text { Left superior } \\
\text { occipital gyrus }\end{array}$ & $(-6,-94,18)$ & 87 & - & - & - & +5.35 & +1.23 & $<0.0001$ & -3.67 & -0.87 & 0.0028 \\
\hline
\end{tabular}

\subsection{Changes in seed-based rsFC}

Significant seed-based rsFC changes across pre-training, post-training, and follow-up sessions were found between the right angular gyrus and the superior occipital gyrus (Fig. 5). A summary of significant rsFC changes is shown in Table 2.

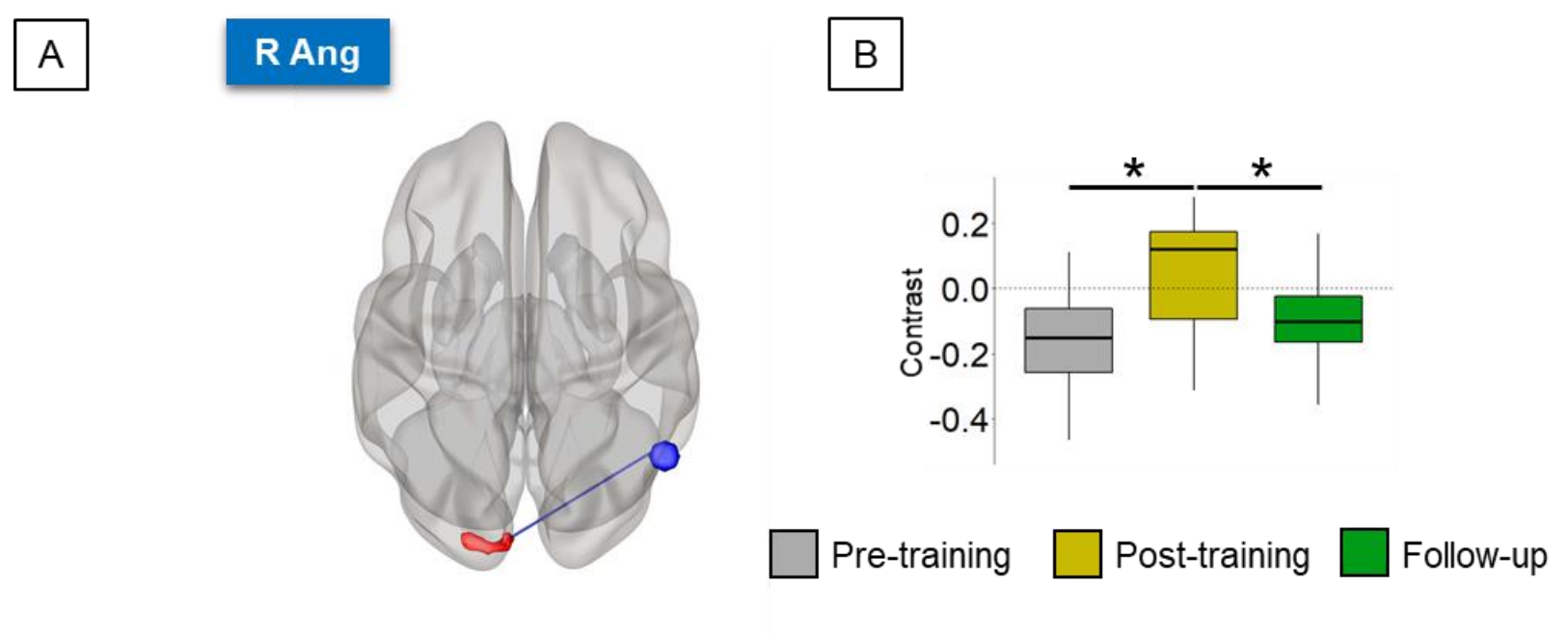

Figure 5. (A) rsFC analyses show that the bilateral middle occipital gyrus presented differences of $F C$ with individual $D M N$ regions across sessions. Blue and red clusters represent DMN regions and seed-based rsFC clusters, respectively, projected onto glass brains in superior view. (B) Boxplots represent contrast values (regulation vs baseline) from the regFC for each session; gray, yellow, and green represent pre-training, post-training, and follow-up sessions, respectively. The dashed black lines in the boxplots represent the zero level. Asterisks represent significant differences corrected for multiple comparisons by the Tukey method $(p<.05)$.

Degree of rsFC significantly changed across pre-training, post-training, and follow-up sessions, according to one-way ANOVAs, in two of the regions provided for neurofeedback training: right IPS $\left(F(2,28)=4.40, p=0.022, \eta^{2}=0.24\right)$ and PCC $\left(F(2,28)=3.86, p=0.03, \eta^{2}=0.22\right)$ (Fig. 6). Post-hoc analyses showed that the degree of rsFC increased in right IPS from pre-training to 
follow-up $\left(\right.$ mean $_{\text {pre-training }}=0.08$, mean $_{\text {post-training }}=0.11, \mathrm{SE}=0.006, \mathrm{t}(28)=2.41, \mathrm{p}=0.03, \mathrm{~d}=0.93$ ) and increased in PCC from post-training to follow-up (mean post-training $=0.07$, mean $_{\text {follow-up }}=0.09$, $\mathrm{SE}=0.006, \mathrm{t}(28)=2.78, \mathrm{p}=0.03, \mathrm{~d}=0.79)$.
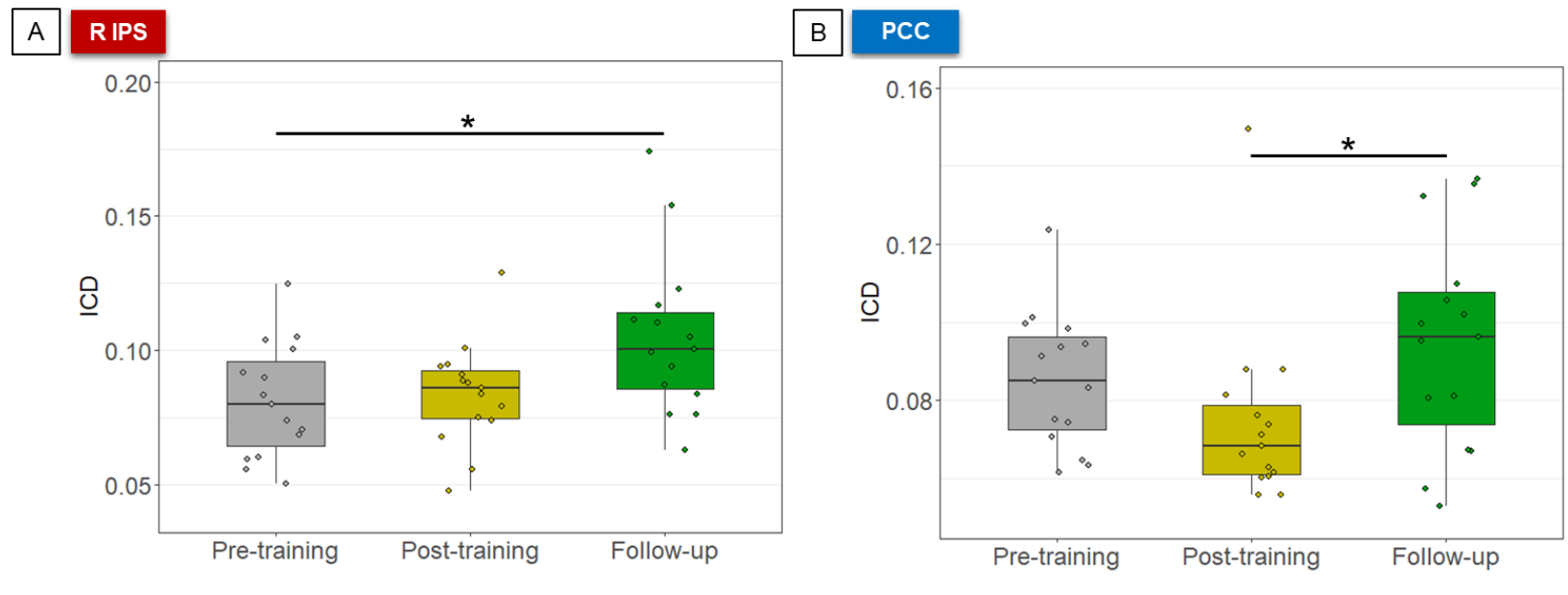

Figure 6. Higher degree of rsFC was observed in the follow-up session (A) for the right IPS compared to the pretraining session and $(B)$ for the PCC compared to the post-training session. Asterisks represent significant differences in post-hoc analyses, corrected for multiple comparisons by the Tukey method $(p<0.05)$. $R$ IPS = right intraparietal sulcus, $P C C=$ posterior cingulate cortex.

\subsection{Behavioral effect and questionnaires}

We first showed that shorter reaction times in early trials of the PVT - i.e., an improvement of sustained attention measured in the first minutes of the test - were associated with the neurofeedback training, as reported in our previous publication (Pamplona et al., 2020a). Yet, this improved ability of sustained attention in early trials of the PVT was statistically lost after two months (Fig. 7). Here, the linear mixed model analysis showed a significant interaction between the factors day and trial $(F(1,5091)=6.58, p=0.0014)$, and according to the post-hoc analysis, the follow-up reaction time in the PVT during early trials was not different from pre-training (t(5091) $=0.66, \mathrm{p}=0.8$; follow-up: $\mathrm{M}=343 \mathrm{~ms}, \mathrm{Cl}=[318,368]$; pre-training: $\mathrm{M}=344 \mathrm{~ms}, \mathrm{Cl}=[320,369]$ ) but it was longer than in the post-training session $(\mathrm{t}(5091)=2.40, \mathrm{p}=0.04$; post-training: $\mathrm{M}=336$ $\mathrm{ms}, \mathrm{Cl}=[311,361])$. Moreover, worsened sustained attention was observed in late trials of the PVT in the follow-up session compared to a pre-training session $(t(5091)=3.60, p=0.0010$; follow-up: $\mathrm{M}=361 \mathrm{~ms}, \mathrm{Cl}=[336,386]$; pre-training: $\mathrm{M}=351 \mathrm{~ms}, \mathrm{Cl}=[326,376])$, but not different compared to a post-training session $(\mathrm{t}(5091)=2.07, \mathrm{p}=0.10 ; \mathrm{M}=356 \mathrm{~ms}, \mathrm{Cl}=[331,381])$. Hypothesized or exploratory attentional states during follow-up transfer regulation were not 
different from pre-training sessions (all FDR-corr. ps > 0.05). A list of strategies used for regulation and baseline blocks during pre-training, post-training, and follow-up transfer sessions is shown in Table S2.

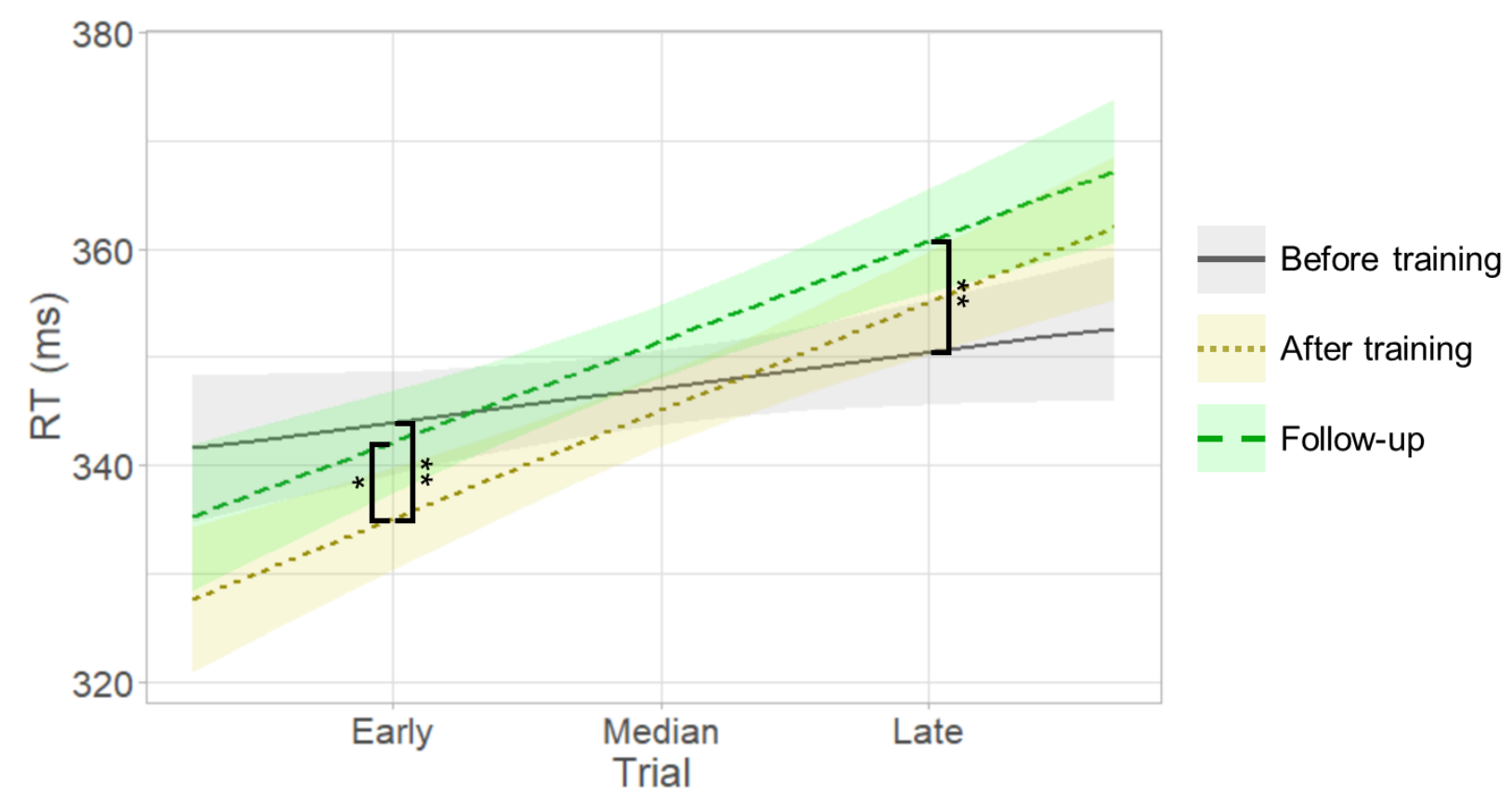

Figure 7. Decreased response times in early stages of the PVT, observed directly after the training, returned to pretraining levels in the follow-up session. The follow-up session also shows a greater detriment in the performance in late trials, as compared to pre-training levels. Reaction time (RT) is shown as a function of trial number in the PVT, which reflects performance changes over time. Gray, yellow, and green colors represent measurements in pre-training, posttraining, and follow-up sessions, respectively. Asterisks represent significant differences in post-hoc tests following corrected for multiple comparisons using the Tukey correction ( $\left.{ }^{* *} p<0.01,{ }^{*} p<0.05\right)$.

\section{DISCUSSION}

In this study, we investigated brain and behavioral changes associated with network-based fMRI neurofeedback training for sustained attention using transfer and resting-state runs conducted before, one day after, and two months after neurofeedback training. In the long term, the following effects were observed: 
- Participants were still able to upregulate the differential activity between predefined SAN and DMN, related to the simultaneous up-modulation of externally-oriented attention and down-regulation of internally-oriented cognition, an ability acquired during neurofeedback training.

- Persistent or further improved self-regulation of differential activity SAN minus DMN was driven by the DMN and the right IPS (part of the SAN).

- The training-induced increase in the functional coupling between DMN and occipital cortex during transfer runs was maintained.

- Brain regions in which activity was successfully modulated after neurofeedback training increased their degree of FC in resting-state runs.

On the other hand, some neurofeedback training effects did not persist at follow-up, returning to pre-training levels. In the behavioral level, the improved sustained attention performance after neurofeedback training returned to baseline level two months later. Regarding neural aspects, the training-induced increase in the functional coupling between DMN and occipital cortex returned to baseline in follow-up resting-state runs.

\subsection{Persistent training effects on self-regulation of functional network activity}

Learned ability to self-regulate the differential activity SAN minus DMN using neurofeedback training was still present two months after training. This result corroborates previous research that showed that acquired self-regulation skills can be maintained over the course of months (Amano et al., 2016; Rance et al., 2018; Robineau et al., 2017). In our study, we observed maintained control over the differential activity SAN minus DMN after a relatively short learning phase (i.e., two sessions of $45 \mathrm{~min}$ each), and that this skill can be retained over an extended period of time (i.e., at least two months).

This acquired control was mainly driven by changes in the DMN (Figs. 2 and 3). While for the PCC a similarly training-induced enhancement of down-regulation of PCC in post-training and follow-up sessions was observed (Fig. 2C), improved down-regulation of mPFC and angular gyrus was observed only after two months and not immediately after training (Figs. $2 \mathrm{C}$ and 3 ). This DMN down-regulation improvement in the long term, initiated by neurofeedback training, is in line with previous arguments (Rance et al., 2018). The authors argued that, once subjects learn control over brain activity via neurofeedback, they can continue practicing self-regulation and integrating it into life habits, which might lead to continuing improvement and neural 
reorganization. Specifically in our study, continued improvement in DMN down-regulation suggests that participants became more effective in suspending intrusive thoughts, detrimental to sustained attention (Weissman et al., 2006), in the long term. Since activation in the DMN is associated with internally oriented attention (Bonnelle et al., 2011; Gusnard et al., 2001; Hinds et al., 2013; Mason et al., 2007), improving DMN down-regulation over time might reflect a reduced proneness to mind-wandering in favor of externally oriented attention. Therefore, as intended, participants learned and maintained the ability of modulating the interaction between large-scale networks (SAN and DMN), which might have implications for the ability of censoring spontaneous task-irrelevant thoughts that affect externally oriented cognition.

The training-induced ability to up-regulate the right IPS in transfer runs was maintained after two months (Fig. 2C). The IPS is part of the DAN and the activation within this network was present during regulation periods throughout transfer runs, even pre-training ones (Fig. 3). Activation in this network is related to the preparation and execution of top-down or goal-directed attention (Corbetta and Shulman, 2002; Fox et al., 2005). Maintained long-term voluntary activation of IPS might indicate a sustained ability in recruiting important neural resources mediating top-down attention. Observed activation in the DAN in all sessions might have been a consequence of measuring intact top-down attention in healthy participants. Since the participants received instructions about regulation strategies from the beginning and the task had a top-down attention component, they could activate this network without difficulty. Yet, on top of this existing ability, the training provided them with the maintained ability of enhanced IPS upregulation.

We observed that the occipital gyrus was less activated in post-training and follow-up sessions compared to pre-training (Figs. 3 and S2). This region is coupled with the DAN (Yeo et al., 2011) and lower activation of this region is related to the suppression or reduction of processing of visual information during the regulation periods (Benedek et al., 2016). Moreover, we observed that the thalamus was activated in the follow-up session during regulation (Fig. 3). Thalamic activity can be related to general arousal maintaining alertness (Sarter et al., 2001). Thalamic activity during vigilant attention decreases over time but bounces back when new conditions are imposed, exerting a role in compensatory attentional effort (Langner and Eickhoff, 2013). As we observed thalamic activity during self-regulation requested after a long period after training, we deduce that the thalamus was involved in additional arousal to compensate for a less automatic state of sustained attention, compared to the post-training session. 


\subsection{Short- and long-term regFC and rsFC changes due to neurofeedback training}

We observed that our neurofeedback training induced regFC and rsFC changes in both short and long terms (Table 2). Previous literature reported changes in rsFC due to neurofeedback training in clinical (Scheinost et al., 2013; Yuan et al., 2014) and healthy samples (Megumi et al., 2015; Zhang et al., 2013). As argued by Rance and colleagues, changes in FC may be continuously reinforced over time after neurofeedback training (Rance et al., 2018), based on previous findings of continuously altered FC after neurofeedback training over the course of days (Harmelech et al., 2013), weeks (Yuan et al., 2014), or months (Megumi et al., 2015). Our findings corroborate this concept in the order of months.

In seed-to-voxel analyses, we observed increased FC between the DMN and the occipital cortex (Figs. 4 and 5) directly after neurofeedback training. Despite other significant seed-based regFC were found between SAN and angular gyrus, postcentral gyrus, and hippocampus (Fig. S3), we here focus on the recurrent regFC and rsFC findings between DMN and occipital cortex. Similar to a study from Benedek and colleagues, this increased FC might reflect an improved top-down mechanism suppressing internally generated distracting thoughts initiated by sensory stimulation (Benedek et al., 2016). However, in our study, there was a crucial difference between regFC (Fig. 4) and rsFC changes (Fig. 5): while FC increased during transfer runs directly after training and in the follow-up sessions, it only increased directly after training and returned to baseline in the long term for resting-state runs. This might indicate that the subjects can actively bolster this functional coupling in the long term upon request, but the transferability to situations unrelated to self-regulation is limited to the short term only. This may reverberate with our findings for behavioral changes being limited to the short term only (further discussed below). This way, improved FC between the DMN and occipital cortex might be a marker of an improved ability in suppressing irrelevant thoughts in situations independent of explicit performing of self-regulation.

The degree of rsFC changed specifically for successfully modulated regions through neurofeedback training in the long term (Fig. $2 \mathrm{C}$ and Fig. 6). Since we used a voxel-wise analysis approach that allows for mapping the degree of FC in the whole brain (Scheinost et al., 2012), this result provides evidence that neurofeedback training may alter the degree of $\mathrm{FC}$ between a region and other regions of the brain. In line with Megumi et al., 2015, our findings reinforce the link between neurofeedback modulation and alterations observed in resting-state (Megumi et al., 2015). These long-term changes in the degree of FC might represent integrative processes underlying plasticity related to the acquired skill. Because these differences were observed in the 
long term, we conclude that the degree of $\mathrm{FC}$ might need an effective regulation learning combined with a relatively long period after intervention so that the plasticity is observed (reflecting the Hebbian principle (Hebb, 1949)).

\subsection{Network-based fMRI neurofeedback training for sustained attention leads to transient behavioral effects}

The improvement in sustained attention, induced in the short term by the proposed neurofeedback training, was not maintained after two months (Fig. 7). Our results are distinct from previous studies that reported persistent effects or even continued improvement in the long term after neurofeedback interventions (Amano et al., 2016; Cortese et al., 2017; Rance et al., 2018; Robineau et al., 2017). Rance and colleagues argued that this continued improvement would likely be a common temporal pattern of behavioral modulation regardless of the targeted brain region. However, not every neurofeedback study exhibits persistent behavioral changes. For example, similar to our results, an EEG-based neurofeedback study aimed for reducing nicotine addiction reported that the short-term changes in symptom reduction were followed by a gradual return toward the baseline in the long term (Bu et al., 2019). The reason why some studies report persistence or continuing improvement of behavioral effects while others report a long-term return to the baseline still needs to be clarified. We conjecture that, if the cognitive aspect to be modulated with neurofeedback is intact or highly optimized in a population, behavioral changes would be of a small magnitude due to ceiling effects and the ability is transferred only in a transient manner, not persistent in the long term. Furthermore, we suggest that long-lasting neurofeedbackinduced regulation skills might be in some cases dissociated from enduring behavioral effects, which may speak against the transferability to real-life consequences of some neurofeedback interventions.

We also found transient effects in rsFC changes due to neurofeedback. Similar to regFC changes, there was increased rsFC between the DMN and occipital cortex in the post- compared to the pre-training session. However, while reg FC continued increasing in the long term, rsFC returned to the baseline in the long term. We conjecture that the persistence of behavioral effects might depend on plasticity (observed independently of self-regulation, like at rest) supporting sustained attention. Further studies are necessary to establish the relationship between long-term functional neuroplasticity and behavioral effects due to neurofeedback training. If this conjecture is valid, 
rsFC could serve as an indicator of persistent behavioral changes or clinical symptoms and transferability of neurofeedback effects into real-life situations.

\subsection{Recommendation of follow-up measurements in neurofeedback studies}

As there is accrued evidence demonstrating that neurofeedback training leads to long-lasting effects (Amano et al., 2016; Cortese et al., 2017; Megumi et al., 2015; Rance et al., 2018; Robineau et al., 2017), we would like to comment on the importance of including follow-up measurements in neurofeedback studies and give recommendations based on our findings. First, whenever possible, one should include assessments of functional or anatomical plasticity to assess brain alterations in the long term due to the training, rather than only shortly after intervention or only behavioral or regulation-specific measurements. Although still in the MR environment, resting-state measurements are independent of regulation runs and might reflect transfer effects of neurofeedback training. Brain imaging follow-up measurements may help indicate neural reshaping over time after completed interventions (Robineau et al., 2017). If neurofeedback-induced effects continue increasing over time, measuring them only shortly after a training intervention may lead to undervalued results (Rance et al., 2018) or to limited assumptions about the transferability of behavioral effects. Follow-up sessions may also help consolidate neuroscientific theories using neurofeedback as a causal intervention (Sulzer et al., 2013b) and help define biomarkers as targets for neurotherapy (Yamada et al., 2017). Second, since it is desired that a given intervention converts practice into enduring effects in a clinical setting, follow-up assessments could demonstrate that the proposed neurofeedback approach would be a more relevant option for therapy. Therefore, neurofeedback studies addressing symptoms in a clinical population should always count on follow-up assessments. Third, we observed that, despite clear long-lasting effects in terms of neural self-regulation, persistent behavioral changes can sometimes be dissociated from brain findings (Sitaram et al., 2017). Therefore, follow-up assessments of behavioral effects should also be performed whenever possible. This may raise questions about the utility of a proposed neurofeedback approach for modulating behavior or symptoms in an out-of-scanner scenario, the strategic choice of sensitive psychometric instruments, and the characterization of the targeted population. Fourth, we argue that neurofeedback training and resting-state/psychometric acquisitions should be made on different days whenever possible, since sleep has an important role in consolidating learning and producing lasting changes in the brain (Walker and Stickgold, 2004). Fifth, our experiment provides evidence that short sessions are enough (two training sessions of $45 \mathrm{~min}$ in separate 
days, in our study) for producing long-term effects (Rance et al., 2018), in terms of brain activity regulation and connectivity.

\subsection{Limitations}

The main limitation of this study is that the follow-up assessments were not controlled by applications in another group submitted to similar conditions. Although behavioral effects in the first study were controlled by the inclusion of another group that performed the psychometric tests outside the scanner only (without training), the present study would require another group receiving sham feedback inside the scanner in similar periods of time to be properly controlled. Therefore, we cannot assert that the findings (such as the ones involving FC) are entirely a product of neurofeedback training, as they may contain the influence of time of experiment, habituation to the MR environment, fatigue, among others. However, evidence in favor of the specificity of the findings is the fact that post-training self-regulation results were reproduced in the long term.

Furthermore, the sample size is modest. Although expensive, there is a trend for higher sample sizes in fMRI-based neurofeedback studies (Fede et al., 2020). Nevertheless, non-parametric statistics, such as TFCE for statistical mapping, were used to adequately detect activity changes in our moderately sized sample. Another limitation was the partially missing questionnaire data for assessments of attentional states and traits, reducing the sample size in the post-training session substantially and underpowering results related to conscious attentional aspects. Moreover, the follow-up results due to the proposed neurofeedback training of sustained attention refer to a period of two months; therefore, the discussion about follow-up effects should be limited to this period.

\subsection{Conclusion}

Our findings indicate a long-term brain-behavior dissociation for the proposed neurofeedback training approach: although learning to control the differential activity SAN minus DMN might have increased sustained attention in the short term, maintaining the regulation ability was not sufficient to observe persistent training-induced behavioral changes. We argue that, as participants learned to control the activity between large-scale networks, this would lead to changes in the correlational structure of these networks. These maintained functional changes were observed only upon selfregulation, but not independent of it. We therefore conjecture that favorable brain states (i.e., 
regulation of associated activity and connectivity), as induced by our training, may be sufficient but not necessary preconditions for cognitive changes. Although the reasons for the long-term dissociation between training and behavioral effects are unclear, specific rsFC might be a marker and a necessary condition for long-term behavioral effects.

Since neurofeedback studies vary substantially in design and outcomes, this work encourages researchers to include, whenever possible, follow-up sessions for a comprehensive evaluation of study-specific effects, including multiple measurements for a complete, time-resolved depiction of the effects. This might be especially important in clinical settings, where long-lasting effects after interventions are desirable to maintain mitigation of symptoms.

\section{Acknowledgements}

We thank Prof. Dustin Scheinost for providing technical advice on intrinsic connectivity distribution, Dr. Alfonso Nieto-Castanon for support with CONN software, Dr. Philipp Stämpfli for technical support with imaging, and Prof. Christian Gaser for advice on the TFCE technique. This work was supported by the Brazilian National Council for Scientific and Technological Development (CNPq), the Brazilian National Council for the Improvement of Higher Education (CAPES), the Swiss National Science Foundation (BSSG10_155915, 100014_178841, 32003B_166566, and PP00P1_170506/1), the Foundation for Research in Science and the Humanities at the University of Zurich (STWF-17-012), the Baugarten Stiftung, and the Swiss Government. All authors declare no conflict of interest.

\section{REFERENCES}

Amano, K., Shibata, K., Kawato, M., Sasaki, Y., Watanabe, T., 2016. Learning to associate orientation with color in early visual areas by associative decoded fMRI neurofeedback. Curr. Biol. 26, 1861-1866. https://doi.org/10.1016/j.cub.2016.05.014

Anderson, K., Deane, K., Lindley, D., Loucks, B., Veach, E., 2012. The effects of time of day and practice on cognitive abilities: The PEBL Tower of London, Trail-making, and Switcher tasks. Pebl Tech. Rep. 1-5.

Andrews-Hanna, J.R., Smallwood, J., Spreng, R.N., 2014. The default network and self-generated thought: Component processes, dynamic control, and clinical relevance. Ann. N. Y. Acad. Sci. 1316, 29-52. https://doi.org/10.1111/nyas. 12360 
Behzadi, Y., Restom, K., Liau, J., Liu, T.T., 2007. A component based noise correction method (CompCor) for BOLD and perfusion based fMRI. Neuroimage 37, 90-101. https://doi.org/10.1016/j.neuroimage.2007.04.042

Benedek, M., Jauk, E., Beaty, R.E., Fink, A., Koschutnig, K., Neubauer, A.C., 2016. Brain mechanisms associated with internally directed attention and self-generated thought. Sci. Rep. 6, 22959.

https://doi.org/10.1038/srep22959

Bonnelle, V., Leech, R., Kinnunen, K.M., Ham, T.E., Beckmann, C.F., De Boissezon, X., Greenwood, R.J., Sharp, D.J., 2011. Default mode network connectivity predicts sustained attention deficits after traumatic brain injury. J. Neurosci. 31, 13442-13451. https://doi.org/10.1523/JNEUROSCI.1163-11.2011

Brett, M., Anton, J.-L., Valabregue, R., Poline, J.-B., 2002. Region of interest analysis using an SPM toolbox, in: 8th International Conference on Functional Mapping of the Human Brain. Sendai, p. 497.

Broadbent, D.E., Cooper, P.F., FitzGerald, P., Parkes, K.R., 1982. The Cognitive Failures Questionnaire (CFQ) and its correlates. Br. J. Clin. Psychol. 21, 1-16. https://doi.org/10.1111/j.2044-8260.1982.tb01421.x

Bu, J., Young, K.D., Hong, W., Ma, R., Song, H., Wang, Y., Zhang, W., Hampson, M., Hendler, T., Zhang, X., 2019. Effect of deactivation of activity patterns related to smoking cue reactivity on nicotine addiction. Brain 142, 1827-1841. https://doi.org/10.1093/brain/awz114

Cohen, J., Cohen, P., 1983. Applied multiple regression/correlation analysis for the behavioral sciences. Taylor \& Francis Group. https://doi.org/10.4324/9780203774441

Conners, K.K., Epstein, J.N., Angold, A., Klaric, J., 2003. Continuous performance test performance in a normative epidemiological sample. J. Abnorm. Child Psychol. 31, 555-562. https://doi.org/10.1023/A:1025457300409

Corbetta, M., Shulman, G.L., 2002. Control of goal-directed and stimulus-driven attention in the brain. Nat. Rev. Neurosci. 3, 201-215. https://doi.org/10.1038/nrn755

Cortese, A., Amano, K., Koizumi, A., Lau, H., Kawato, M., 2017. Decoded fMRI neurofeedback can induce bidirectional confidence changes within single participants. Neuroimage 149, 323-337. https://doi.org/10.1016/j.neuroimage.2017.01.069

deBettencourt, M.T., Cohen, J.D., Lee, R.F., Norman, K.A., Turk-Browne, N.B., D Cohen, J., Lee, R.F., A Norman, K., B Turk-Browne, N., 2015. Closed-loop training of attention with real-time brain imaging. Nat. Neurosci. 18, 470478. https://doi.org/10.1038/nn.3940

Dinges, D.F., Powell, J.W., 1985. Microcomputer analyses of performance on a portable, simple visual RT task during sustained operations. Behav. Res. Methods, Instruments, Comput. 17, 652-655. https://doi.org/10.3758/BF03200977

Fan, J., McCandliss, B.D., Sommer, T., Raz, A., Posner, M.I., 2002. Testing the efficiency and independence of attentional networks. J. Cogn. Neurosci. 14, 340-347. https://doi.org/10.1162/089892902317361886

Fede, S.J., Dean, S.F., Manuweera, T., Momenan, R., 2020. A guide to literature informed decisions in the design of real time fMRI neurofeedback studies: A systematic review. Front. Hum. Neurosci.

https://doi.org/10.3389/fnhum.2020.00060 
Fox, M.D., Snyder, A.Z., Vincent, J.L., Corbetta, M., Van Essen, D.C., Raichle, M.E., 2005. The human brain is intrinsically organized into dynamic, anticorrelated functional networks. Proc. Natl. Acad. Sci. 102, 9673-9678. https://doi.org/10.1073/pnas.0504136102

Gusnard, D.A., Akbudak, E., Shulman, G.L., Raichle, M.E., 2001. Medial prefrontal cortex and self-referential mental activity: Relation to a default mode of brain function. Proc. Natl. Acad. Sci. 98, 4259-4264. https://doi.org/10.1073/pnas.071043098

Harmelech, T., Friedman, D., Malach, R., 2015. Differential magnetic resonance neurofeedback modulations across extrinsic (Visual) and intrinsic (default-mode) nodes of the human cortex. J. Neurosci. 35, 2588-2595. https://doi.org/10.1523/jneurosci.3098-14.2015

Harmelech, T., Preminger, S., Wertman, E., Malach, R., 2013. The day-after effect: long term, Hebbian-like restructuring of resting-state $\mathrm{fMRI}$ patterns induced by a single epoch of cortical activation. J. Neurosci. 33, 9488-9497. https://doi.org/10.1523/JNEUROSCI.5911-12.2013

Harris, I.M., Egan, G.F., Sonkkila, C., Tochon-Danguy, H.J., Paxinos, G., Watson, J.D.G., 2000. Selective right parietal lobe activation during mental rotation: A parametric PET study. Brain 123, 65-73. https://doi.org/10.1093/brain/123.1.65

Haugg, A., Sladky, R., Skouras, S., McDonald, A., Craddock, C., Kirschner, M., Herdener, M., Koush, Y., Papoutsi, M., Keynan, J.N., Hendler, T., Cohen Kadosh, K., Zich, C., Maclnnes, J., Adcock, R.A., Dickerson, K., Chen, N.-K., Young, K., Bodurka, J., Yao, S., Becker, B., Auer, T., Schweizer, R., Pamplona, G.S.P., Emmert, K., Haller, S., Van De Ville, D., Blefari, M.-L., Kim, D.-Y., Lee, J.-H., Marins, T., Fukuda, M., Sorger, B., Kamp, T., Liew, S.-L., Veit, R., Spetter, M., Weiskopf, N., Scharnowski, F., 2020. Can we predict real-time fMRI neurofeedback learning success from pretraining brain activity? Hum. Brain Mapp. 41, 3839-3854. https://doi.org/10.1002/hbm.25089

Hebb, D., 1949. The Organization of Behavior: A Neuropsychological Theory.

Helton, W.S., 2004. Validation of a short stress state questionnaire. Proc. Hum. Factors Ergon. Soc. Annu. Meet. 48, 1238-1242. https://doi.org/10.1177/154193120404801107

Henson, R.N., 2015. Analysis of variance (ANOVA), in: Brain Mapping: An Encyclopedic Reference. Elsevier, pp. 477-481.

Hinds, O., Ghosh, S., Thompson, T.W., Yoo, J.J., Whitfield-Gabrieli, S., Triantafyllou, C., Gabrieli, J.D.E., 2011. Computing moment-to-moment BOLD activation for real-time neurofeedback. Neuroimage 54, 361-368. https://doi.org/10.1016/j.neuroimage.2010.07.060

Hinds, O., Thompson, T.W., Ghosh, S., Yoo, J.J., Whitfield-Gabrieli, S., Triantafyllou, C., Gabrieli, J.D.E., 2013. Roles of default-mode network and supplementary motor area in human vigilance performance: evidence from realtime fMRI. J. Neurophysiol. 109, 1250-1258. https://doi.org/10.1152/jn.00533.2011

Kim, H.-C., Tegethoff, M., Meinlschmidt, G., Stalujanis, E., Belardi, A., Jo, S., Lee, J., Kim, D.-Y., Yoo, S.-S., Lee, J.H., 2019. Mediation analysis of triple networks revealed functional feature of mindfulness from real-time fMRI neurofeedback. Neuroimage 195, 409-432. https://doi.org/10.1016/j.neuroimage.2019.03.066 
Koush, Y., Ashburner, J., Prilepin, E., Sladky, R., Zeidman, P., Bibikov, S., Scharnowski, F., Nikonorov, A., De Ville, D. Van, 2017a. OpenNFT: An open-source Python/Matlab framework for real-time fMRI neurofeedback training based on activity, connectivity and multivariate pattern analysis. Neuroimage 156, 489-503. https://doi.org/10.1016/j.neuroimage.2017.06.039

Koush, Y., Meskaldji, D.E., Pichon, S., Rey, G., Rieger, S.W., Linden, D.E.J., Van De Ville, D., Vuilleumier, P., Scharnowski, F., 2017b. Learning control over emotion networks through connectivity-based neurofeedback. Cereb. Cortex 27, 1193-1202. https://doi.org/10.1093/cercor/bhv311

Krause, F., Kogias, N., Krentz, M., Lührs, M., Goebel, R., Hermans, E.J., 2021. Self-regulation of stress-related largescale brain network balance using real-time fMRI Neurofeedback. Neuroimage. https://doi.org/10.1016/j.neuroimage.2021.118527

Langner, R., Eickhoff, S.B., 2013. Sustaining attention to simple tasks: A meta-analytic review of the neural mechanisms of vigilant attention. Psychol. Bull. 139, 870-900. https://doi.org/10.1037/a0030694

Lawrence, N.S., Ross, T.J., Hoffmann, R., Garavan, H., Stein, E.A., 2003. Multiple neuronal networks mediate sustained attention. J. Cogn. Neurosci. 15, 1028-1038. https://doi.org/10.1162/089892903770007416

Linden, D.E.J., Habes, I., Johnston, S.J., Linden, S., Tatineni, R., Subramanian, L., Sorger, B., Healy, D., Goebel, R., 2012. Real-time self-regulation of emotion networks in patients with depression. PLoS One 7, 1-10. https://doi.org/10.1371/journal.pone.0038115

Mason, M.F., Norton, M.I., Van Horn, J.D., Wegner, D.M., Grafton, S.T., Macrae, C.N., 2007. Wandering minds: The default network and stimulus-independent thought. Science (80-. ). 315, 393-395. https://doi.org/10.1126/science.1131295

McLaren, D.G., Ries, M.L., Xu, G., Johnson, S.C., 2012. A generalized form of context-dependent psychophysiological interactions (gPPI): a comparison to standard approaches. Neuroimage 61, 1277-1286. https://doi.org/10.1016/j.neuroimage.2012.03.068

Megumi, F., Yamashita, A., Kawato, M., Imamizu, H., 2015. Functional MRI neurofeedback training on connectivity between two regions induces long-lasting changes in intrinsic functional network. Front. Hum. Neurosci. 9. https://doi.org/10.3389/fnhum.2015.00160

Mehler, D.M.A., Sokunbi, M.O., Habes, I., Barawi, K., Subramanian, L., Range, M., Evans, J., Hood, K., Lührs, M., Keedwell, P., Goebel, R., Linden, D.E.J., 2018. Targeting the affective brain-a randomized controlled trial of real-time fMRI neurofeedback in patients with depression. Neuropsychopharmacology 43, 2578-2585. https://doi.org/10.1038/s41386-018-0126-5

Mueller, S.T., Piper, B.J., 2014. The Psychology Experiment Building Language (PEBL) and PEBL Test Battery. J. Neurosci. Methods 222, 250-259. https://doi.org/10.1016/j.jneumeth.2013.10.024

O'Reilly, J.X., Woolrich, M.W., Behrens, T.E.J., Smith, S.M., Johansen-Berg, H., 2012. Tools of the trade: Psychophysiological interactions and functional connectivity. Soc. Cogn. Affect. Neurosci. 7, 604-609. https://doi.org/10.1093/scan/nss055 
Pamplona, G.S.P., Heldner, J., Langner, R., Koush, Y., Michels, L., Ionta, S., Scharnowski, F., Salmon, C.E.G., 2020a. Network-based fMRI-neurofeedback training of sustained attention. Neuroimage 221, 117194. https://doi.org/10.1016/j.neuroimage.2020.117194

Pamplona, G.S.P., Vieira, B.H., Scharnowski, F., Salmon, C.E.G., 2020b. Personode: A toolbox for ICA map classification and individualized ROI definition. Neuroinformatics 18, 339-349. https://doi.org/10.1007/s12021019-09449-4

Raichle, M.E., MacLeod, A.M., Snyder, A.Z., Powers, W.J., Gusnard, D.A., Shulman, G.L., 2001. A default mode of brain function. Proc. Natl. Acad. Sci. 98, 676-682. https://doi.org/10.1073/pnas.98.2.676

Ramot, M., Kimmich, S., Gonzalez-Castillo, J., Roopchansingh, V., Popal, H., White, E., Gotts, S.J., Martin, A., 2017. Direct modulation of aberrant brain network connectivity through real-time NeuroFeedback. Elife 6, e28974. https://doi.org/10.7554/eLife.28974

Rance, M., Walsh, C., Sukhodolsky, D.G., Pittman, B., Qiu, M., Kichuk, S.A., Wasylink, S., Koller, W.N., Bloch, M., Gruner, P., Scheinost, D., Pittenger, C., Hampson, M., 2018. Time course of clinical change following neurofeedback. Neuroimage 181, 807-813. https://doi.org/10.1016/j.neuroimage.2018.05.001

Robineau, F., Meskaldji, D.E., Koush, Y., Rieger, S.W., Mermoud, C., Morgenthaler, S., Van De Ville, D., Vuilleumier, P., Scharnowski, F., 2017. Maintenance of voluntary self-regulation learned through real-time fMRI neurofeedback. Front. Hum. Neurosci. 11, 131. https://doi.org/10.3389/fnhum.2017.00131

Rubinov, M., Sporns, O., 2010. Complex network measures of brain connectivity: Uses and interpretations. Neuroimage 52, 1059-1069. https://doi.org/10.1016/J.NEUROIMAGE.2009.10.003

Sarter, M., Givens, B., Bruno, J.P., 2001. The cognitive neuroscience of sustained attention: where top-down meets bottom-up. Brain Res. Brain Res. Rev. 35, 146-160. https://doi.org/10.1016/s0165-0173(01)00044-3

Scheinost, D., Benjamin, J., Lacadie, C.M., Vohr, B., Schneider, K.C., Ment, L.R., Papademetris, X., Constable, R.T., 2012. The intrinsic connectivity distribution: A novel contrast measure reflecting voxel level functional connectivity. Neuroimage 62, 1510-1519. https://doi.org/10.1016/j.neuroimage.2012.05.073

Scheinost, D., Hsu, T.W., Avery, E.W., Hampson, M., Constable, R.T., Chun, M.M., Rosenberg, M.D., 2020. Connectome-based neurofeedback: A pilot study to improve sustained attention. Neuroimage 212, 116684. https://doi.org/10.1016/j.neuroimage.2020.116684

Scheinost, D., Stoica, T., Saksa, J., Papademetris, X., Constable, R.T., Pittenger, C., Hampson, M., 2013. Orbitofrontal cortex neurofeedback produces lasting changes in contamination anxiety and resting-state connectivity. Transl. Psychiatry 3, e250. https://doi.org/10.1038/tp.2013.24

Shepard, R.N., Metzler, J., 1971. Mental rotation of three-dimensional objects. Science (80-. ). 171, 701-703.

Sitaram, R., Ros, T., Stoeckel, L., Haller, S., Scharnowski, F., Lewis-Peacock, J., Weiskopf, N., Blefari, M.L., Rana, M., Oblak, E., Birbaumer, N., Sulzer, J., 2017. Closed-loop brain training: The science of neurofeedback. Nat. Rev. Neurosci. 18, 86-100. https://doi.org/10.1038/nrn.2016.164

Smith, S.M., Nichols, T.E., 2009. Threshold-free cluster enhancement: Addressing problems of smoothing, threshold 
dependence and localisation in cluster inference. Neuroimage 44, 83-98.

https://doi.org/10.1016/j.neuroimage.2008.03.061

Spreng, R., 2012. The fallacy of a "task-negative" network. Front. Psychol. 3, 145.

https://doi.org/10.3389/fpsyg.2012.00145

Spunt, B., 2016. spunt/bspmview: BSPMVIEW v.20161108. https://doi.org/10.5281/ZENODO.168074

Sulzer, J., Haller, S., Scharnowski, F., Weiskopf, N., Birbaumer, N., Blefari, M.L., Bruehl, A.B., Cohen, L.G., DeCharms, R.C., Gassert, R., Goebel, R., Herwig, U., LaConte, S., Linden, D., Luft, A., Seifritz, E., Sitaram, R., 2013a. Real-time fMRI neurofeedback: Progress and challenges. Neuroimage 76, 386-399.

https://doi.org/10.1016/j.neuroimage.2013.03.033

Sulzer, J., Sitaram, R., Blefari, M.L., Kollias, S., Birbaumer, N., Stephan, K.E., Luft, A., Gassert, R., 2013b. Neurofeedback-mediated self-regulation of the dopaminergic midbrain. Neuroimage 83, 817-825. https://doi.org/10.1016/j.neuroimage.2013.05.115

Thompson, G.J., Magnuson, M.E., Merritt, M.D., Schwarb, H., Pan, W.J., Mckinley, A., Tripp, L.D., Schumacher, E.H., Keilholz, S.D., 2013. Short-time windows of correlation between large-scale functional brain networks predict vigilance intraindividually and interindividually. Hum. Brain Mapp. 34, 3280-3298. https://doi.org/10.1002/hbm.22140

Walker, M.P., Stickgold, R., 2004. Sleep-dependent learning and memory consolidation. Neuron 44, 121-133. https://doi.org/https://doi.org/10.1016/j.neuron.2004.08.031

Watanabe, T., Sasaki, Y., Shibata, K., Kawato, M., 2017. Advances in fMRI real-time neurofeedback. Trends Cogn. Sci. 21, 997-1010. https://doi.org/10.1016/j.tics.2017.09.010

Weissman, D.H., Roberts, K.C., Visscher, K.M., Woldorff, M.G., 2006. The neural bases of momentary lapses in attention. Nat. Neurosci. 9, 971-978. https://doi.org/10.1038/nn1727

West, S.G., Aiken, L.S., Krull, J.L., 1996. Experimental personality designs: Analyzing categorical by continuous variable interactions. J. Pers. 64, 1-48. https://doi.org/10.1111/j.1467-6494.1996.tb00813.x

Whitfield-Gabrieli, S., Nieto-Castanon, A., 2012. Conn: A functional connectivity toolbox for correlated and anticorrelated brain networks. Brain Connect. 2, 125-141. https://doi.org/10.1089/brain.2012.0073

Worsley, K.J., Marrett, S., Neelin, P., Vandal, A.C., Friston, K.J., Evans, A.C., 1996. A unified statistical approach for determining significant signals in images of cerebral activation. Hum. Brain Mapp. 4, 58-73. https://doi.org/10.1002/(SICI)1097-0193(1996)4:1<58::AID-HBM4>3.0.CO;2-O

Yamada, T., Hashimoto, R.-I., Yahata, N., Ichikawa, N., Yoshihara, Y., Okamoto, Y., Kato, N., Takahashi, H., Kawato, M., 2017. Resting-state functional connectivity-based biomarkers and functional MRI-based neurofeedback for psychiatric disorders: A challenge for developing theranostic biomarkers. Int. J. Neuropsychopharmacol. 20, 769-781. https://doi.org/10.1093/ijnp/pyx059

Yeo, B.T., Krienen, F.M., Sepulcre, J., Sabuncu, M.R., Lashkari, D., Hollinshead, M., Roffman, J.L., Smoller, J.W., Zöllei, L., Polimeni, J.R., Fischl, B., Liu, H., Buckner, R.L., 2011. The organization of the human cerebral cortex 
estimated by intrinsic functional connectivity. J. Neurophysiol. 106, 1125-1165.

https://doi.org/10.1152/jn.00338.2011

Yuan, H., Young, K.D., Phillips, R., Zotev, V., Misaki, M., Bodurka, J., 2014. Resting-state functional connectivity modulation and sustained changes after real-time functional magnetic resonance imaging neurofeedback training in depression. Brain Connect. 4, 690-701. https://doi.org/10.1089/brain.2014.0262

Zhang, G., Zhang, H., Li, X., Zhao, X., Yao, L., Long, Z., 2013. Functional alteration of the DMN by learned regulation of the PCC using real-time fMRI. IEEE Trans. Neural Syst. Rehabil. Eng. 21, 595-606.

https://doi.org/10.1109/TNSRE.2012.2221480

Zilverstand, A., Sorger, B., Sarkheil, P., Goebel, R., 2015. fMRI neurofeedback facilitates anxiety regulation in females with spider phobia. Front. Behav. Neurosci. 9, 1-12. https://doi.org/10.3389/fnbeh.2015.00148

Zweerings, J., Hummel, B., Keller, M., Zvyagintsev, M., Schneider, F., Klasen, M., Mathiak, K., 2019. Neurofeedback of core language network nodes modulates connectivity with the default-mode network: A double-blind fMRI neurofeedback study on auditory verbal hallucinations. Neuroimage 189, 533-542.

https://doi.org/10.1016/j.neuroimage.2019.01.058 